\title{
Rapid isolation and profiling of a diverse panel of human monoclonal antibodies targeting the SARS-CoV-2 spike protein
}

\author{
Seth J. Zost $\mathbb{1}^{1,14}$, Pavlo Gilchuk ${ }^{1,14}$, Rita E. Chen ${ }^{2,3}$, James Brett Case ${ }^{3}$, Joseph X. Reidy ${ }^{1}$, \\ Andrew Trivette ${ }^{1}$, Rachel S. Nargi ${ }^{1}$, Rachel E. Sutton', Naveenchandra Suryadevara', Elaine C. Chen (1) ${ }^{4}$, \\ Elad Binshtein ${ }^{1}{ }^{1}$, Swathi Shrihari $\mathbb{1}^{3}$, Mario Ostrowski ${ }^{5}$, Helen Y. Chu ${ }^{6}$, Jonathan E. Didier ${ }^{7}$, \\ Keith W. MacRenaris7, Taylor Jones', Samuel Day', Luke Myers ${ }^{\mathbb{1} 1}$, F. Eun-Hyung Lee $\mathbb{1}^{8}$, \\ Doan C. Nguyen ${ }^{8}{ }^{8}$, Ignacio Sanz ${ }^{10}{ }^{8}$, David R. Martinez ${ }^{9}$, Paul W. Rothlauf ${ }^{10,11}$, Louis-Marie Bloyet ${ }^{11}$, \\ Sean P. J. Whelan ${ }^{10,11}$, Ralph S. Baric ${ }^{9}$, Larissa B. Thackray $\mathbb{1}^{3}$, Michael S. Diamond ${ }^{2,3,11,12}$, \\ Robert H. Carnahan $1,13 \bowtie$ and James E. Crowe Jr. $1,4,13 \bowtie$
}

\begin{abstract}
Antibodies are a principal determinant of immunity for most RNA viruses and have promise to reduce infection or disease during major epidemics. The novel coronavirus SARS-CoV-2 has caused a global pandemic with millions of infections and hundreds of thousands of deaths to date ${ }^{1,2}$. In response, we used a rapid antibody discovery platform to isolate hundreds of human monoclonal antibodies (mAbs) against the SARS-CoV-2 spike (S) protein. We stratify these mAbs into five major classes on the basis of their reactivity to subdomains of $S$ protein as well as their cross-reactivity to SARS-CoV. Many of these mAbs inhibit infection of authentic SARS-CoV-2 virus, with most neutralizing mAbs recognizing the receptor-binding domain (RBD) of S. This work defines sites of vulnerability on SARS-CoV-2 S and demonstrates the speed and robustness of advanced antibody discovery platforms.
\end{abstract}

Human mAbs against the $\mathrm{S}$ glycoprotein on the viral surface mediate immunity to other betacoronaviruses including SARS-CoV ${ }^{3-7}$ and Middle East respiratory syndrome coronavirus (MERS-CoV) ${ }^{8-17}$. Because of this, we and others have hypothesized that human mAbs may have promise for use in prophylaxis, post-exposure prophylaxis or treatment of SARS-CoV-2 infection ${ }^{18}$. MAbs can neutralize betacoronaviruses by several mechanisms, including blocking attachment of the $\mathrm{S}$ protein receptor-binding domain (RBD) to a receptor on host cells (which, for SARS-CoV and SARS-CoV-2 (ref. ${ }^{1}$ ), is angiotensin-converting enzyme 2 (ACE2) $)^{12}$. We hypothesized that the SARS-CoV-2 S protein would induce diverse human neutralizing antibodies following natural infection. While antibody discovery and therapeutic candidate development usually takes months to years, there is an urgent need to both characterize the human immune response to SARS-CoV-2 infection and to develop potential medical countermeasures. Using Zika virus as a simulated pandemic pathogen and leveraging recent technological advances in synthetic genomics and single-cell sequencing, we recently isolated hundreds of human mAbs from a single-B-cell suspension and tested them in vitro for neutralization and for protection in small animals and non-human primates, all within $78 \mathrm{~d}^{19}$. Using similar methodologies and further efficiency improvements, we sought to obtain human mAbs rapidly for SARS-CoV-2 from the $B$ cells of some of the first humans identified with the infection in North America. We used an approach similar to that in our previous technical demonstration with Zika; however, for the SARS-CoV-2 discovery effort we report here, we used several different workflows in parallel (Fig. 1 and Supplementary Table 1), which we completed in an expedited time frame (Fig. 1).

We first developed or obtained antigens and recombinant proteins necessary for identifying and isolating antigen-reactive $\mathrm{B}$ cells. We synthesized a complementary-DNA encoding a stabilized trimeric prefusion ectodomain of $\mathrm{S}$ protein $\left(\mathrm{S}_{2} \mathrm{P}_{\text {ecto }}\right)^{20}$, expressed the protein in 293-F cells, and verified the presence of the prefusion conformation by electron microscopy (Extended Data Fig. 1 and Supplementary Table 2). We also synthesized and expressed the $S$ protein $\mathrm{RBD}\left(\mathrm{S}_{\mathrm{RBD}}\right)$ and obtained recombinant $\mathrm{S}$ protein amino-terminal domain $\left(\mathrm{S}_{\mathrm{NTD}}\right)$ that had been prepared by academic or commercial sources. Using these tools, we designed a $\mathrm{mAb}$ discovery approach focused on identifying naturally occurring human mAbs specific for $\mathrm{S}$.

We obtained informed consent and collected blood samples from four patients infected in China who were among the earliest identified patients with SARS-CoV-2 infection in North America,

'Vanderbilt Vaccine Center, Vanderbilt University Medical Center, Nashville, TN, USA. ${ }^{2}$ Department of Pathology \& Immunology, Washington University School of Medicine, St. Louis, MO, USA. ${ }^{3}$ Department of Medicine, Washington University School of Medicine, St. Louis, MO, USA. ${ }^{4}$ Department of Pathology, Microbiology, and Immunology, Vanderbilt University Medical Center, Nashville, TN, USA. ${ }^{5}$ Department of Medicine, University of Toronto, Toronto, Ontario, Canada. ' ${ }^{6}$ Division of Allergy and Infectious Diseases, University of Washington, Seattle, WA, USA. 'Berkeley Lights, Inc., Emeryville, CA, USA. ${ }^{8}$ Department of Medicine, Emory University, Atlanta, GA, USA. 'Department of Epidemiology, University of North Carolina at Chapel Hill, Chapel Hill, NC, USA. ${ }^{10}$ Program in Virology, Harvard Medical School, Boston, MA, USA. "'Department of Molecular Microbiology, Washington University School of Medicine, St. Louis, MO, USA. ${ }^{12}$ Andrew M. and Jane M. Bursky Center for Human Immunology and Immunotherapy Programs, Washington University School of Medicine, St. Louis, MO, USA. ${ }^{13}$ Department of Pediatrics, Vanderbilt University Medical Center, Nashville, TN, USA. ${ }^{14}$ These authors contributed

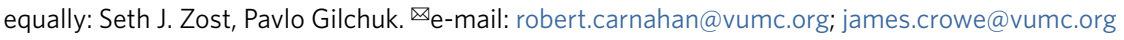




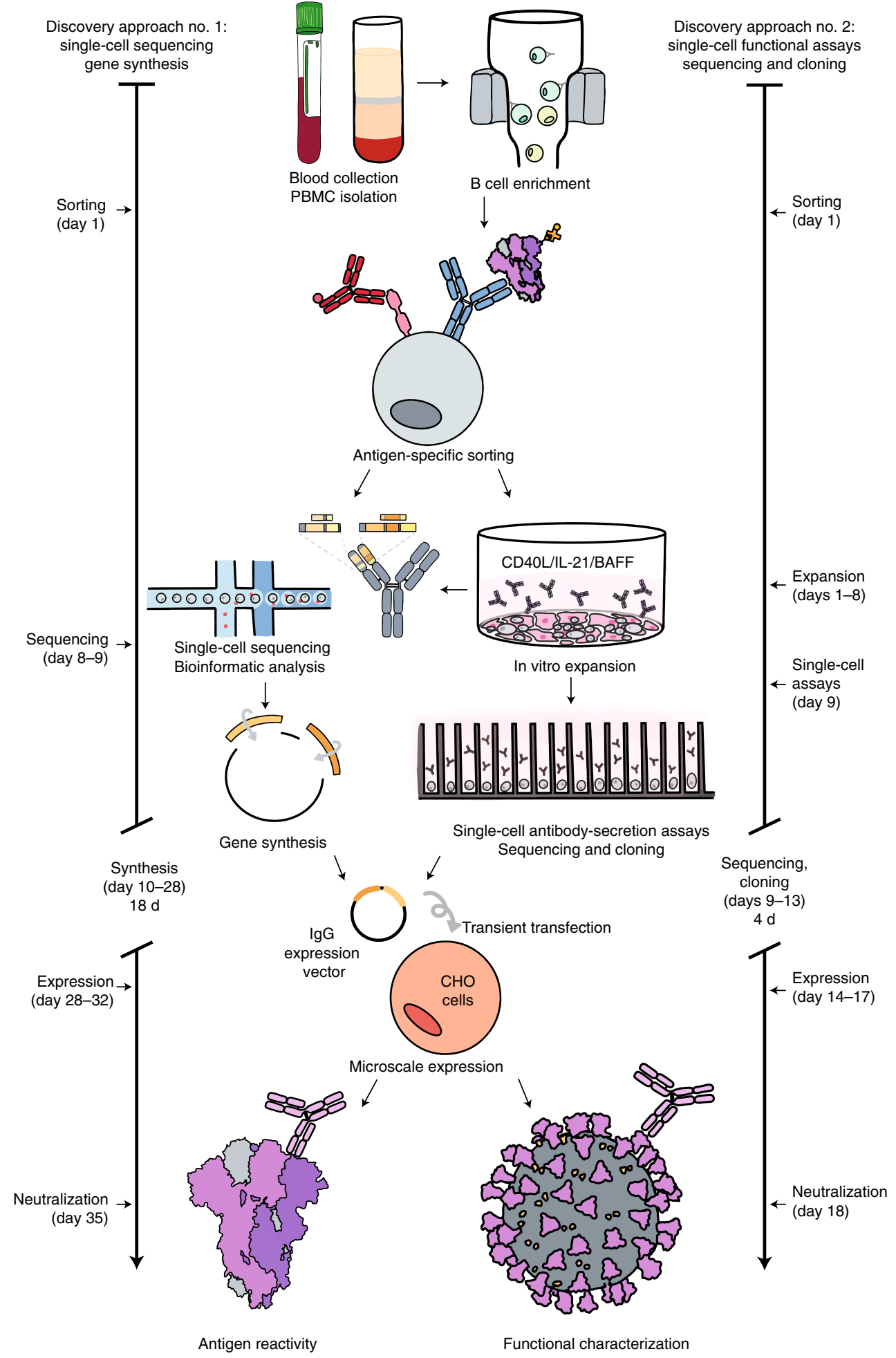

Fig. 1 | Workflows and timelines. a, Overview of rapid $\mathrm{mAb}$ discovery workflows. The overall scheme is shown, representing the several specific workflows conducted in parallel (specified in Supplementary Table 1). Blood was collected and white blood cells were separated, B cells were enriched from PBMCs by negative selection using magnetic beads and antigen-specific cells were isolated by flow-cytometric sorting and then were processed for direct B cell selection and sequencing or in vitro expansion/activation. Cultured B cells were loaded on a Beacon instrument (Berkeley Lights) for functional screening (Extended Data Fig. 3 and Supplementary Video 1) or in a Chromium device (10X Genomics) followed by reverse transcription with $\mathrm{PCR}$, sequence analysis, CDNA gene synthesis and cloning into an expression vector and microscale lgG expression in Chinese hamster ovary $(\mathrm{CHO})$ cells by transient transfection. Recombinant IgG was tested by ELISA for binding to determine antigen reactivity and by a high-throughput neutralization screening assay (xCelligence; ACEA) (Extended Data Fig. 4) with authentic virus in a biosafety-level-3 (BSL-3) laboratory for functional characterization. 


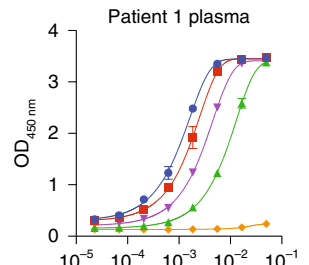

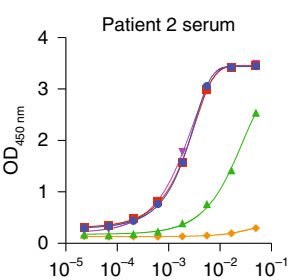

Patient 3 plasma

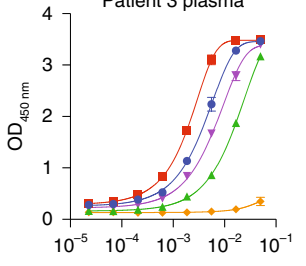

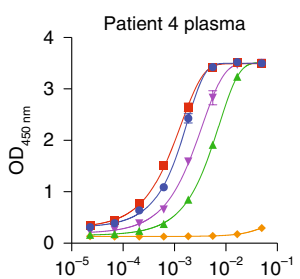

$\checkmark$ SARS-CoV S2P

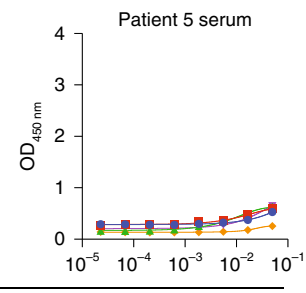

$\simeq$ SARS-CoV-2 S NTD

$\sim$ PBS
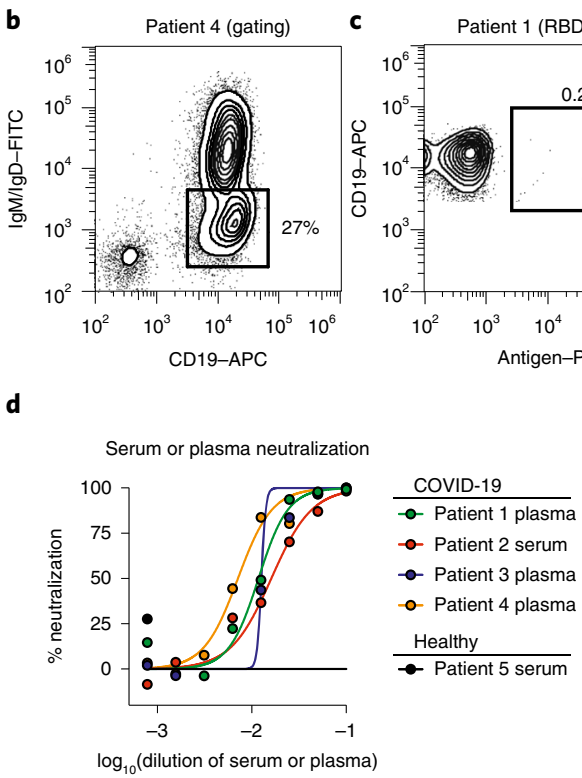

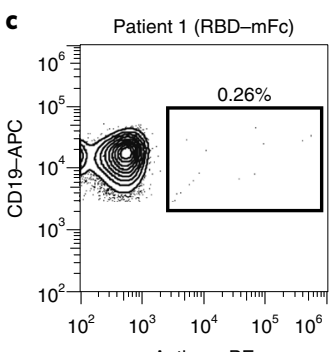

e

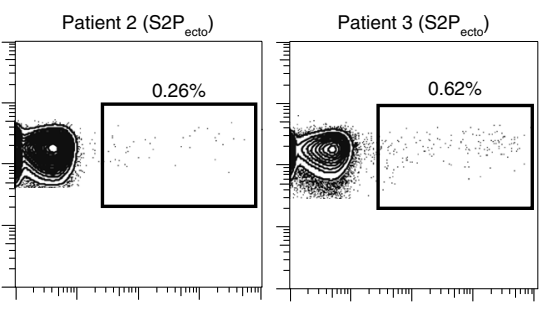

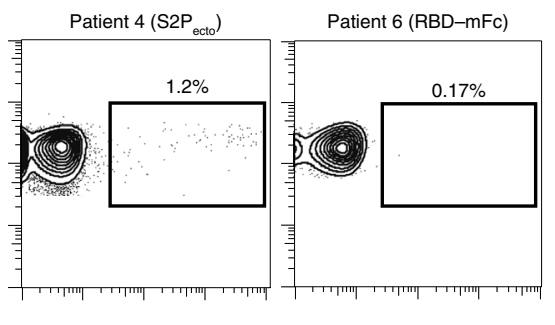

f

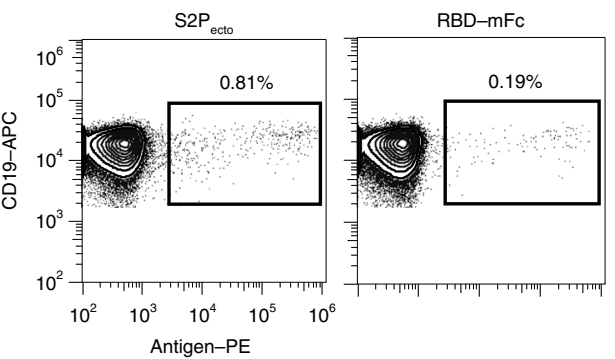

Fig. 2 | Characterization of SARS-CoV-2 immune donor samples. a, Serum or plasma antibody reactivity for the four SARS-CoV-2 exposed patients and one non-immune healthy control, assessed by ELISA using SARS-CoV-2 S2P $\mathrm{P}_{\text {ecto, }} \mathrm{S}_{\mathrm{RBD}}, \mathrm{S}_{\mathrm{NTD}}, \mathrm{SARS}-\mathrm{CoV}$ S2P $\mathrm{P}_{\text {ecto }}$ or PBS. OD 450 , optical density measurement using a microplate reader with a 450-nm filter. b. Gating for memory B cells in total B cells enriched by negative selection using magnetic beads for patient 4; cells were stained with anti-CD19 antibody conjugated to allophycocyanin (APC) and anti-IgM and anti-IgD antibodies conjugated to fluorescein isothiocyanate (FITC). c, Analytical flow cytometric analysis of B cells for patients 1 to 4, compared with that of a healthy individual (patient 6 ). Plots

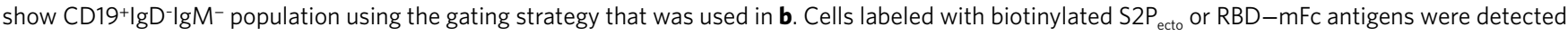
using phycoerythrin (PE)-conjugated streptavidin. d, Plasma or serum neutralizing activity against the WA1/2020 strain SARS-CoV-2 for patients 1 to 4 or a healthy donor (patients 6 ). The percentage neutralization is reported. e, FACS isolation of S2P $P_{\text {ecto }}$ or RBD-mFc-reactive B cells from pooled $B$ cells of patients 3 and 4 . Plots show $C^{-19}{ }^{+} \lg D^{-} \lg M^{-}$population using gating strategy as in $\mathbf{b}$, and antigen-reactive B cells were identified as in $\mathbf{c}$. $\mathbf{f}$, Lymphoblastoid cell line (LCL) supernatant neutralization. Neutralization of the WA1/2020 strain SARS-CoV-2 by supernatant collected from cell cultures of $\mathrm{S}_{\mathrm{P}} \mathrm{P}_{\text {ecto }}$ - or RBD-mFc-sorted memory B cells that had been stimulated in bulk in vitro on feeder layers expressing CD40L and secreting IL-21 and BAFF. The supernatants were tested in a ten-point dilution series in the FRNT, and percentage neutralization is reported. Values shown are the mean \pm s.d. of technical duplicates.

as well as one healthy donor who served as a negative control (Supplementary Table 3). These patients had a history of recent laboratory-confirmed SARS-CoV-2 infection acquired in Wuhan or Beijing, China. The samples were obtained $35 \mathrm{~d}$ (patient 1; the case identified in the United States ${ }^{21}$ ), $36 \mathrm{~d}$ (patient 2) or $50 \mathrm{~d}$ (patients 3 and 4) after the onset of symptoms. We tested plasma or serum specimens from the four patients infected with SARS-CoV-2, or from a healthy donor (patient 5) as control. Serum/plasma antibody ELISA binding assays using $\mathrm{S}_{2} \mathrm{P}_{\text {ecto }}, \mathrm{S}_{\mathrm{RBD}}$ or $\mathrm{S}_{\mathrm{NTD}}$ protein from SARS-CoV-2 or $\mathrm{S}_{2} \mathrm{P}_{\text {ecto }}$ protein from SARS-CoV revealed that the previously infected patients had circulating antibodies that recognized each of the proteins tested, with the highest reactivity against the SARS-CoV-2 S2P $\mathrm{P}_{\text {ecto }}$ and $\mathrm{S}_{\mathrm{RBD}}$ proteins (Fig. 2a). Each of the previously infected patients also had circulating antibodies that bound to SARS-CoV S2P ${ }_{\text {ecto }}$. The serum antibodies from the healthy donor did not react with any of the antigens. B cells were enriched from peripheral blood mononuclear cells (PBMCs) by negative selection using antibody-coated magnetic beads and were stained with phenotyping antibodies specific for CD19, $\operatorname{IgD}$ and $\operatorname{IgM}$. Analytical flow cytometry was performed to assess the frequency of antigen-specific memory B cells for each donor. We identified class-switched memory B cells by gating for an $\operatorname{IgD}^{-} \mathrm{IgM}^{-} \mathrm{CD} 19^{+}$ population (Fig. 2b, and Extended Data Fig. 2). From this memory $B$ cell population, we identified antigen-reactive cells using biotinylated recombinant $\mathrm{S}_{2} \mathrm{P}_{\text {ecto }}$ protein or biotinylated $\mathrm{RBD}$ fused to mouse $\mathrm{Fc}(\mathrm{RBD}-\mathrm{mFc})$. Patients 1 and 2 had very low frequencies of antigen-specific memory $B$ cells that were not greater than twofold above the background staining frequency in a non-immune sample (from patient 6) (Fig. 2c). In contrast to patients 1 and 2, convalescence in patients 3 and 4 was 2 weeks later, and they exhibited $0.62 \%$ or $1.22 \%$ of class-switched B cells that reacted with antigen (Fig. 2c). Patients 3 and 4 also exhibited high titers in a serum-antibody focus reduction neutralization test (FRNT) with an authentic SARS-CoV-2 strain (WA/1/2020) (Fig. 2d). Therefore, we focused subsequent efforts on sorting B cells from the specimens of patients 3 and 4 , which were pooled for efficiency. 


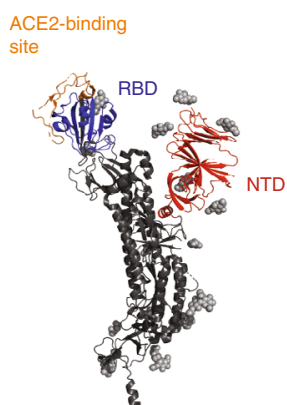

SARS-CoV-2 S

monome

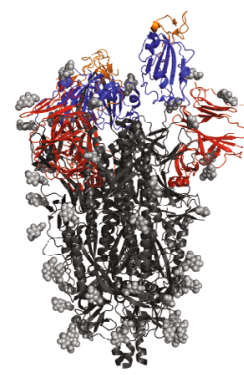

SARS-CoV-2 S

trimer

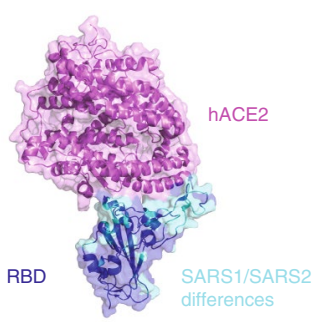

SARS-CoV-2

$\mathrm{S}_{\mathrm{RBD}}-\mathrm{hACE} 2$ b

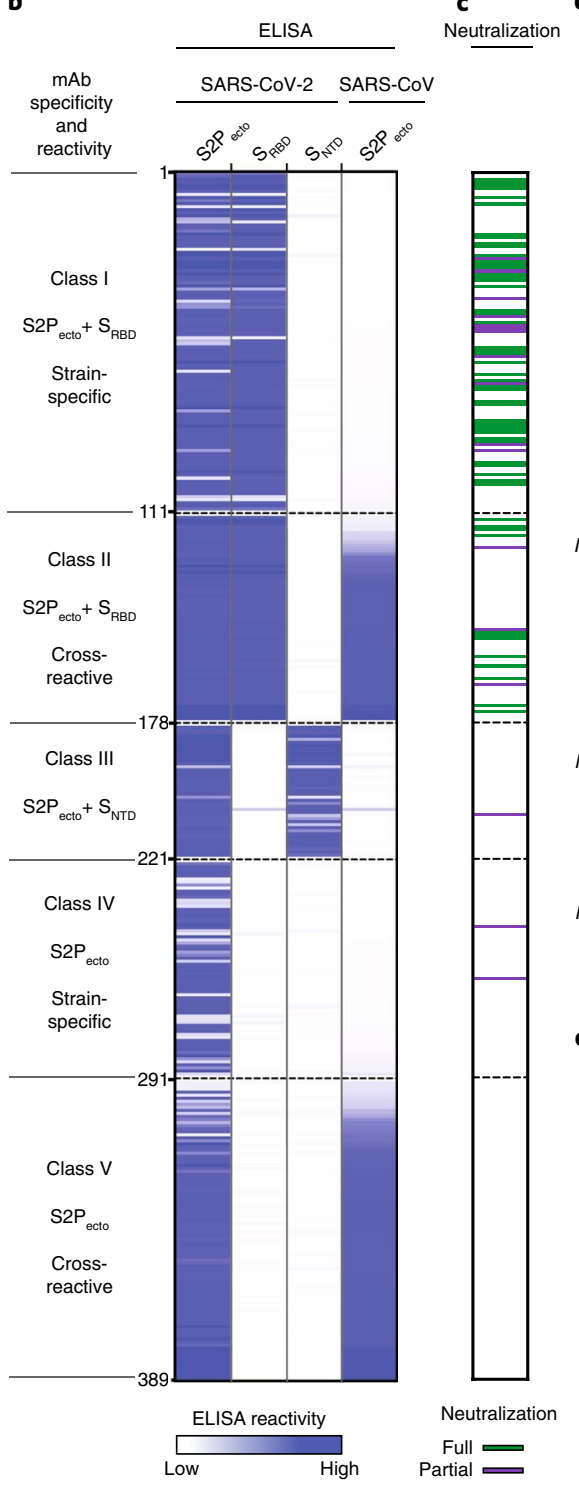

d

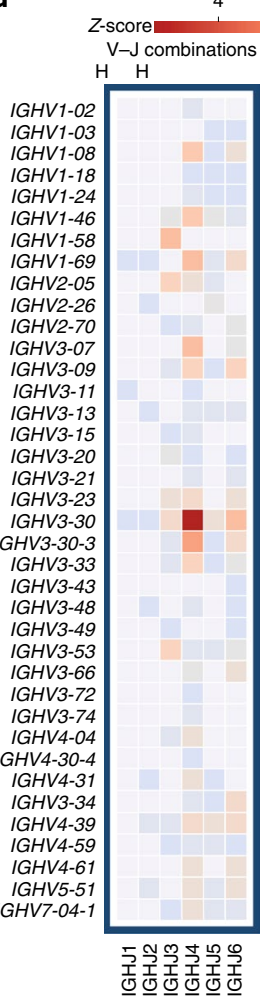

e

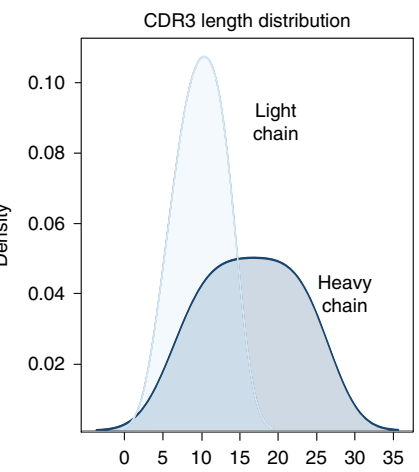

IGLV1-51

IGLV2-08

IGLV2-11

IGLV2-14

IGLV2-23

IGLV3-01

IGLV3-10

IGLV3-19

IGLV3-21

IGLV3-25
IGLV3-27

IGLV4-60

IGLV4-69

IGLV6-57

IGLV7-43

GLV9-49
2

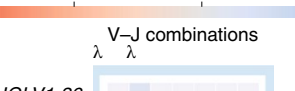

$-2$

$\underset{\kappa}{V}-J_{\kappa}$ combinations

\begin{tabular}{l|l} 
IGLV1-36 & IVKV1-05
\end{tabular}

\begin{tabular}{l|l} 
GLV1-40 & IGKV1-09
\end{tabular}

IGLV1-44 IGKV1-12

IGLV1-50

IGKV1-27

IGKV1-33

IGKV2-28

IGKV2-28

IGKVV-40

GKVVD-29

GKV2D-29

IGKV3-11

IGKV3-20

GKV 3D-20

IGKV3D-20

IGKV $6-21$

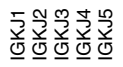

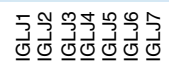

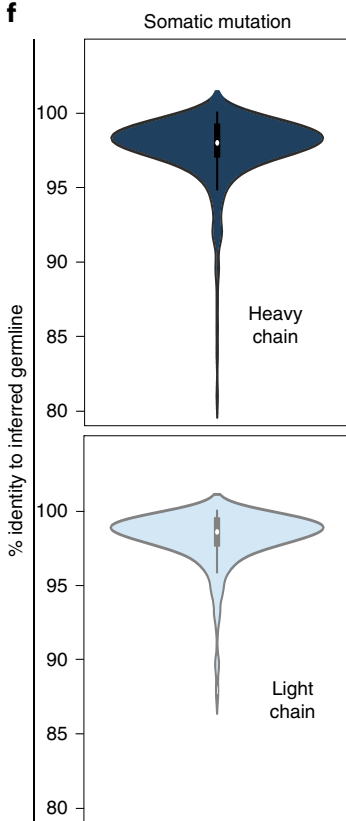

Fig. 3 | Reactivity and functional activity of $\mathbf{3 8 9}$ human mAbs. a, Structures of SARS-CoV-2 S antigen. Top, S protein monomer of SARS-CoV-2 highlighting RBD (blue) and NTD (red) subdomains that were expressed as recombinant proteins. The ACE2-binding site on RBD is shown in orange. Known glycans are shown as light gray spheres (PDB 6VYB) ${ }^{37}$. Middle, The structure of trimeric SARS-CoV-2 spike with one RBD in the 'head up' conformation. Bottom, Structure (PDB 6MOJ) ${ }^{38}$ of SARS-CoV-2 RBD (blue) and hACE2 (pink), highlighting differences between RBDs of SARS-CoV-2 and SARS-CoV (cyan). b, mAbs binding to each of four $\mathrm{S}$ proteins or subdomains. The figure shows a heatmap for binding of $389 \mathrm{mAbs}$ expressed recombinantly, representing OD values collected at $450 \mathrm{~nm}$ for each antigen (range, 0.035-4.5). White indicates a lack of detectable binding, while blue indicates binding and darker blue indicates higher OD values. c, Screening test for neutralizing activity. Each mAb was tested by RTCA neutralization test (Extended Data Figs. 4 and 5) that was based on measurement of rapid cytopathic effect in Vero-furin cells caused by an authentic SARS-CoV-2 (strain WA1/2020) in a BSL-3 laboratory. Green indicates full protection of cells (full neutralization), purple indicates partial protection of cells (partial neutralization) and white indicates that neutralizing activity was not detected. On the basis of both binding and neutralization, we grouped the mAbs

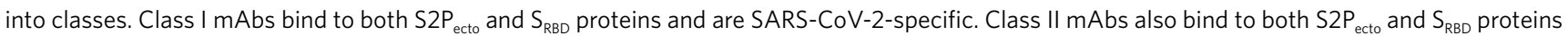

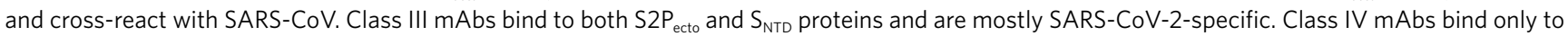
$\mathrm{S} 2 \mathrm{P}_{\text {ecto }}$ protein and are SARS-CoV-2-specific. Class $V \mathrm{mAbs}$ bind only to $\mathrm{S}_{\mathrm{P}}$ ecto protein and cross-react with SARS-CoV. d, Heatmap showing usage of antibody variable-gene segments for variable $(V)$ and joining $(J)$ genes. Of the 389 antibodies tested in $\mathbf{b}$ and $\mathbf{c}, 324$ were found to have unique sequences, and those unique sequences were analyzed for genetic features. The frequency counts are derived from the total number of unique sequences with the corresponding $V$ and $J$ genes. The $V / J$ frequency counts then were transformed into a $Z$-score by first subtracting the average frequency, then normalizing by the s.d. of the set of antigen-reactive mAbs. Red denotes more common gene usage, and blue denotes less common gene usage. e, CDR3 amino acid length distribution. The CDR3 of each sequence was determined using PyIR software. The amino acid length of each CDR3 was counted. The distribution of CDR3 amino acid lengths for heavy or light chains then was plotted as a histogram and fitted using kernel density estimation for the curves. f, Divergence from inferred germline gene sequences. The number of mutations of each mAb relative to the inferred germline variable gene was counted for each clone. These numbers then were transformed into percent values and plotted as violin plots. For the heavy chain, values range from 81 to 100 , with a median of 98 , a 25 th quartile of 97.3 and a 75 th quartile of 99 . For the light chain, values range from 87.5 to 100 , with a median of 98.6 , a 25 th quartile of 97.9 and a 75 th quartile of 99.3. 
a

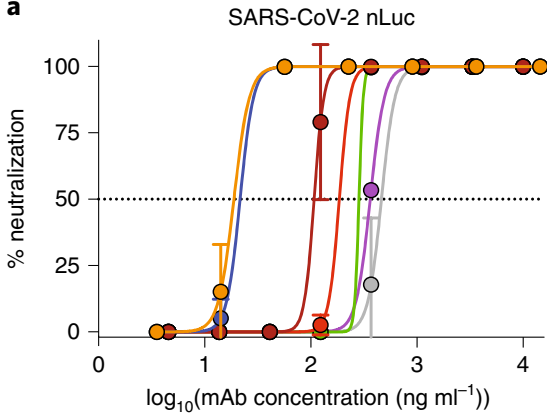

b

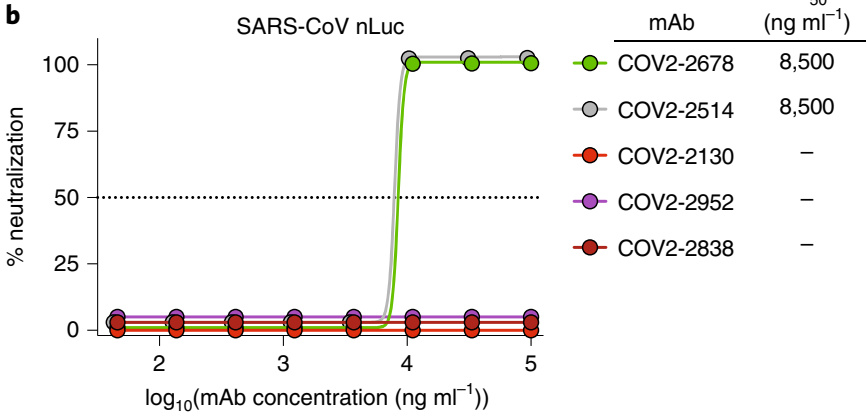

Fig. 4 | Neutralizing activity of potent mAbs against SARS-CoV-2 and SARS-CoV. a, Dose-response neutralization of SARS-CoV-2 luciferase reporter virus by representative potently neutralizing $m A$ bs that were identified by rapid RTCA screening assay. $I C_{50}$ values are indicated for each $\mathrm{mAb}$. Data shown are the mean of two technical replicates from one of two independent experiments, and error bars denote the s.d. for each point. b, Neutralization of SARS-CoV luciferase reporter virus by cross-reactive mAbs COV2-2678 and COV2-2514. IC 50 values are indicated for each mAb, with the '-' symbol indicating that mAbs that did not neutralize SARS-CoV. Data shown are the mean \pm s.d. of two technical replicates from one of two independent experiments.

The pooled memory B cell suspension had frequencies for $\mathrm{S}_{2} \mathrm{P}_{\text {ecto }}$ and $\mathrm{RBD}-\mathrm{mFc}$ that were $0.81 \%$ and $0.19 \%$ of the $\mathrm{IgD}^{-} \mathrm{IgM}^{-} \mathrm{CD} 19^{+}$ population, respectively (Fig. 2e). The bulk sorted $\mathrm{S}_{2} \mathrm{P}_{\text {ecto }}-$ or $\mathrm{RBD}-$ $\mathrm{mFc}$-specific B cells were stimulated on a feeder layer with CD40L, interleukin-21 (IL-21) and B cell activating factor (BAFF) ${ }^{22}$, and the secreted antibodies in the resulting cell-culture supernatants exhibited neutralizing activity against the WA1/2020 strain (Fig. 2f). After $7 \mathrm{~d}$ in culture, these activated B cells were removed from the feeder layers. Roughly half of these B cells were single-cell sequenced using a 10x Chromium single-cell encapsulation automated system, and antibody genes were synthesized as previously described ${ }^{19}$. The remaining cells were loaded onto a Berkeley Lights Beacon optofluidic instrument in a novel medium promoting plasma-cell survival, and antigen reactivity of secreted antibodies from individual B cells was measured for thousands of cells (Extended Data Fig. 3). This single-cell analysis showed that $~ 55 \%$ of cultured B cells secreted SP $2_{\text {ecto }}$-reactive IgG (Supplementary Video 1). Using the Beacon instrument, we also screened a subset of expanded B cells for the ability to block human ACE2 (hACE2) receptor binding to the $\mathrm{RBD}-\mathrm{mFc}$ construct and identified several clones secreting $\mathrm{mAbs}$ that inhibited RBD-hACE2 interaction (Extended Data Fig. 3d). Antigen-reactive B cells were exported from the instrument, and the heavy- and light-chain genes from single B cells were sequenced and cloned into immunoglobulin expression vectors. From 288 total exported cells, we succeeded in cloning 78 antigen-reactive mAbs (Supplementary Table 1), including two closely related mAbs that blocked hACE2 binding to the RBD.
Using the parallel workflows, we isolated 389 recombinant SARS-CoV-2-reactive human mAbs that expressed sufficiently well as recombinant IgG to characterize the activity of the $\mathrm{mAb}$. The recombinant $\mathrm{mAbs}$ were tested for binding in ELISA to recombinant monomeric $\mathrm{S}_{\mathrm{RBD}}$ or $\mathrm{S}_{\mathrm{NTD}}$ of SARS-CoV-2 or trimeric $\mathrm{S}_{2} \mathrm{P}_{\text {ecto }}$ proteins of SARS-CoV-2 or SARS-CoV (Fig. 3a). Neutralizing activity of $\mathrm{mAbs}$ was assessed using an automated real-time cell analysis (RTCA) rapid screening assay ${ }^{19}$ that allows quantitation of cytopathic effect (CPE) with WA1/2020 strain SARS-CoV-2 in Vero-furin cells (Extended Data Fig. 4). The ELISA and neutralization screening assays revealed that the antibodies could be grouped into five binding patterns on the basis of domain recognition and cross-reactivity (Fig. 3b). There were 178 that mAbs recognized the RBD domain and 43 that recognized the NTD domain. Comparison of binding patterns with full or partial neutralizing activity measured by RTCA with SARS-CoV-2 (Fig. 3c) clearly showed that most of the neutralizing antibodies (67/70) mapped to the RBD, revealing the RBD to be the principal site of vulnerability for SARS-CoV-2 neutralization in these patients. Overall, $49 \%$ of the SARS-CoV-2-specific RBD mAbs were neutralizing, and $19 \%$ of the SARS-CoV cross-reactive mAbs were neutralizing. We then used the RTCA assay to rank mAbs by their neutralizing potency (Extended Data Fig. 5) and identified several mAbs with half-maximal inhibitory concentration $\left(\mathrm{IC}_{50}\right.$ ) values below $100 \mathrm{ng}$ $\mathrm{ml}^{-1}$. We examined the sequences for the 389 antibodies to assess the diversity of antigen-specific $\mathrm{B}$ cell clonotypes discovered. The analysis showed that among the $389 \mathrm{mAbs}, 321$ unique amino acid sequences were present and 313 unique $\mathrm{VH}-\mathrm{JH}-\mathrm{CDRH} 3-\mathrm{VL}-\mathrm{JL}-$ CDRL3 clonotypes were represented, with diverse usage of antibody variable genes (Fig. $3 \mathrm{~d}$ and Supplementary Table 4). The length distributions of CDR3 amino acids in the heavy and light chains were typical of human repertoires (Fig. 3e) ${ }^{23}$. The high relatedness of sequences to the inferred germline variable genes observed for this panel of antibodies (Fig. 3f) contrasts with the much higher levels of somatic mutation seen in B cell recall responses against common human pathogens such as influenza ${ }^{24}$. These data suggest that the SARS-CoV-2 antibodies were likely induced during the primary response to SARS-CoV-2 infection and not by a recall response to a distantly related seasonal coronavirus. We next performed neutralization assays with a recombinant vesicular stomatitis virus (VSV) expressing SARS-CoV-2 S (VSV-SARS-CoV-2) ${ }^{25}$ that confirmed neutralizing activity of $49 \mathrm{mAbs}$ identified as neutralizing by the rapid RTCA screening assay (Extended Data Fig. 6). We also confirmed high neutralizing potency for representative potent $\mathrm{mAbs}$ in a quantitative conventional neutralization assay using a SARS-CoV-2 luciferase reporter virus (Fig. 4a). In addition, we tested SARS-CoV S2 $\mathrm{P}_{\text {ecto }}$ cross-reactive mAbs for cross-neutralization of a SARS-CoV luciferase reporter virus (Fig. $4 \mathrm{~b}$ ), which revealed relatively weak neutralization of heterologous SARS-CoV. Together, these results confirmed that $\mathrm{mAbs}$ recognizing multiple epitopes on $\mathrm{S}$ were able to neutralize SARS-CoV-2 and cross-react with SARS-CoV, with most neutralizing $\mathrm{mAbs}$ specific for the RBD of $\mathrm{S}$.

Since the emergence of SARS-CoV-2, several groups have reported the isolation of neutralizing antibodies from survivors that target the S protein ${ }^{26-33}$. There are similarities between our approaches and those of others, for example employing antigen-specific enrichment with both $\operatorname{RBD}^{26,27,29,30,32}$ and full-length $S$ trimer ${ }^{26,28,33}$ as well as the use of single-cell sequencing and gene synthesis ${ }^{29}$. A common finding across these studies is that the SARS-CoV-2 RBD is the target of potent neutralizing antibodies. In this work, we coupled single-B-cell antibody gene sequencing with high-throughput IgG isolation and screening assays to isolate and profile a large number of neutralizing mAbs in a period of only weeks after sample acquisition. The technological advances of our workflow included rapid antigen binding and functional (hACE2 blocking) screens at the single-B-cell level as well as high-throughput (hundreds 
to thousands) screening of mAbs for neutralization of authentic SARS-CoV-2 virus to rapidly identify the leads. In our particular example, sequences of confirmed neutralizing antibodies were transferred to downstream manufacturing partners only $18 \mathrm{~d}$ after antigen-specific cell sorting. However, given the need for affinity maturation and the development of a mature B cell response, there are limits on the timeline from infection to isolation of potent neutralizing antibodies with therapeutic promise. It has been previously shown for Ebola virus infection that potently neutralizing antibodies are not easily isolated from memory B cells until later timepoints in the first year after infection ${ }^{34,35}$. It is possible that our success in identifying antigen-specific B cells from patients 3 and 4 here at 50 $\mathrm{d}$ after onset, but not from patients 1 and 2 at 35 or $36 \mathrm{~d}$ after onset, reflected additional maturation of the memory $\mathrm{B}$ cell response that occurred in the additional 2 weeks of convalescence, although we note that others have isolated neutralizing $\mathrm{mAbs}$ from individuals approximately $30 \mathrm{~d}$ after infection ${ }^{27}$. Overall, our work illustrates the promise of integrating recent technological advances for antibody discovery and helps to define the RBD of SARS-CoV-2 S as a major site of vulnerability for vaccine design and therapeutic-antibody development. The most potent neutralizing human mAbs isolated here also could serve as candidate biologics to prevent or treat SARS-CoV-2 infection ${ }^{36}$.

\section{Online content}

Any methods, additional references, Nature Research reporting summaries, source data, extended data, supplementary information, acknowledgements, peer review information; details of author contributions and competing interests; and statements of data and code availability are available at https://doi.org/10.1038/ s41591-020-0998-X.

Received: 12 May 2020; Accepted: 26 June 2020;

Published online: 10 July 2020

\section{References}

1. Zhou, P. et al. A pneumonia outbreak associated with a new coronavirus of probable bat origin. Nature 579, 270-273 (2020).

2. Zhu, N. et al. A novel coronavirus from patients with pneumonia in China, 2019. N. Engl. J. Med. 382, 727-733 (2020).

3. Sui, J. et al. Potent neutralization of severe acute respiratory syndrome (SARS) coronavirus by a human $\mathrm{mAb}$ to $\mathrm{S} 1$ protein that blocks receptor association. Proc. Natl Acad. Sci. USA 101, 2536-2541 (2004).

4. ter Meulen, J. et al. Human monoclonal antibody as prophylaxis for SARS coronavirus infection in ferrets. Lancet 363, 2139-2141 (2004).

5. ter Meulen, J. et al. Human monoclonal antibody combination against SARS coronavirus: synergy and coverage of escape mutants. PLoS Med. 3, e237 (2006).

6. Zhu, Z. et al. Potent cross-reactive neutralization of SARS coronavirus isolates by human monoclonal antibodies. Proc. Natl Acad. Sci. USA 104, 12123-12128 (2007).

7. Rockx, B. et al. Structural basis for potent cross-neutralizing human monoclonal antibody protection against lethal human and zoonotic severe acute respiratory syndrome coronavirus challenge. J. Virol. 82, 3220-3235 (2008).

8. Chen, Z. et al. Human neutralizing monoclonal antibody inhibition of Middle East respiratory syndrome coronavirus replication in the common marmoset. J. Infect. Dis. 215, 1807-1815 (2017).

9. Choi, J. H. et al. Characterization of a human monoclonal antibody generated from a B-cell specific for a prefusion-stabilized spike protein of Middle East respiratory syndrome coronavirus. PLoS ONE 15, e0232757 (2020).

10. Niu, P. et al. Ultrapotent human neutralizing antibody repertoires against Middle East respiratory syndrome coronavirus from a recovered patient. J. Infect. Dis. 218, 1249-1260 (2018).

11. Wang, L., et al. Importance of neutralizing monoclonal antibodies targeting multiple antigenic sites on the Middle East respiratory syndrome coronavirus spike glycoprotein to avoid neutralization escape. J. Virol. 92, e02002-17 (2018).
12. Wang, N. et al. Structural definition of a neutralization-sensitive epitope on the MERS-CoV S1-NTD. Cell Rep. 28, e3396 (2019).

13. Zhang, S. et al. Structural definition of a unique neutralization epitope on the receptor-binding domain of MERS-CoV spike glycoprotein. Cell Rep. 24, 441-452 (2018).

14. Corti, D. et al. Prophylactic and postexposure efficacy of a potent human monoclonal antibody against MERS coronavirus. Proc. Natl Acad. Sci. USA 112, 10473-10478 (2015).

15. Jiang, L. et al. Potent neutralization of MERS-CoV by human neutralizing monoclonal antibodies to the viral spike glycoprotein. Sci. Transl. Med. 6, 234ra259 (2014).

16. Tang, X. C. et al. Identification of human neutralizing antibodies against MERS-CoV and their role in virus adaptive evolution. Proc. Natl Acad. Sci. USA 111, E2018-E2026 (2014).

17. Ying, T. et al. Exceptionally potent neutralization of Middle East respiratory syndrome coronavirus by human monoclonal antibodies. J. Virol. 88, 7796-7805 (2014).

18. Jiang, S., Hillyer, C. \& Du, L. Neutralizing antibodies against SARS-CoV-2 and other human coronaviruses. Trends Immunol. 41, 355-359 (2020).

19. Gilchuk, P. et al. Integrated technology platform for accelerated discovery of antiviral antibody therapeutics. Nat. Biomed. Eng. (in the press).

20. Wrapp, D. et al. Cryo-EM structure of the 2019-nCoV spike in the prefusion conformation. Science 367, 1260-1263 (2020).

21. Holshue, M. L. et al. First case of 2019 novel coronavirus in the United States. N. Engl. J. Med. 382, 929-936 (2020).

22. Gilchuk, P. et al. Analysis of a therapeutic antibody cocktail reveals determinants for cooperative and broad ebolavirus meutralization. Immunity 52, e312 (2020)

23. Soto, C. et al. High frequency of shared clonotypes in human B cell receptor repertoires. Nature 566, 398-402 (2019)

24. Wrammert, J. et al. Broadly cross-reactive antibodies dominate the human B cell response against 2009 pandemic H1N1 influenza virus infection. J. Exp. Med. 208, 181-193 (2011).

25. Case, J. B. et al. Neutralizing antibody and soluble ACE2 inhibition of a replication-competent VSV-SARS-CoV-2 and a clinical isolate of SARS-CoV-2. Cell Host Microbe https://doi.org/10.1016/j.chom.2020.06.021 (2020).

26. Rogers, T. F. et al. Rapid isolation of potent SARS-CoV-2 neutralizing antibodies and protection in a small animal model. Science https://doi. org/10.1126/science.abc7520 (2020).

27. Robbiani, D. F. et al. Convergent antibody responses to SARS-CoV-2 infection in convalescent individuals. Nature https://doi.org/10.1038/ s41586-020-2456-9 (2020).

28. Brouwer, P. J. M. et al. Potent neutralizing antibodies from COVID-19 patients define multiple targets of vulnerability. Science eabc5902 (2020).

29. Cao, Y. et al. Potent neutralizing antibodies against SARS-CoV-2 identified by high-throughput single-cell sequencing of convalescent patients' B cells. Cell https://doi.org/10.1016/j.cell.2020.05.025 (2020).

30. Shi, R. et al. A human neutralizing antibody targets the receptor binding site of SARS-CoV-2. Nature https://doi.org/10.1038/s41586-020-2381-y (2020).

31. $\mathrm{Wu}, \mathrm{Y}$. et al. A noncompeting pair of human neutralizing antibodies block COVID-19 virus binding to its receptor ACE2. Science 368, 1274-1278 (2020).

32. Ju, B. et al. Human neutralizing antibodies elicited by SARS-CoV-2 infection. Nature https://doi.org/10.1038/s41586-020-2380-z (2020).

33. Wec, A. Z. et al. Broad neutralization of SARS-related viruses by human monoclonal antibodies. Science https://doi.org/10.1126/science.abc7424 (2020).

34. Williamson, L. E. et al. Early human B cell response to Ebola virus in four U.S. survivors of infection. J. Virol. 93, e01439-18 (2019).

35. Davis, C. W. et al. Longitudinal analysis of the human B cell response to Ebola virus infection. Cell 177, e1517 (2019).

36. Zost, S. J. et al. Potently neutralizing human antibodies that block SARS-CoV-2 receptor binding and protect animals. Nature (in the press).

37. Walls, A. C. et al. Structure, function, and antigenicity of the SARS-CoV-2 spike glycoprotein. Cell 181, e286 (2020).

38. Lan, J. et al. Structure of the SARS-CoV-2 spike receptor-binding domain bound to the ACE2 receptor. Nature 581, 215-220 (2020).

Publisher's note Springer Nature remains neutral with regard to jurisdictional claims in published maps and institutional affiliations.

๑ The Author(s), under exclusive licence to Springer Nature America, Inc. 2020 


\section{Methods}

Research participants. We studied four patients in North America with recent laboratory-confirmed symptomatic SARS-CoV-2 infections that were acquired in China (Supplementary Table 3). The studies were approved by the Institutional Review Board of Vanderbilt University Medical Center, and subsite studies were approved by the Institutional Review Board of the University of Washington or the Research Ethics Board of the University of Toronto. Samples were obtained after written informed consent. Patient 1 (35-year-old male) was the earliest reported case of SARS-CoV-2 infection in the United States, who presented with disease in Seattle, Washington, on 19 January 2020 (ref. ${ }^{21}$ ), a blood sample was obtained for study on 19 February 2020. Patient 2 (52-year-old female) was infected following close exposure in Beijing, China, to an infected person from Wuhan, China, during the period between 23 January 2020 to 29 January 2020. She presented with mild respiratory disease symptoms from 1 February 2020 to 4 February 2020 that occurred after travel to Madison, Wisconsin. She obtained a diagnosis of infection by testing at the US Centers for Disease Control on 5 February 2020. Blood samples were obtained for study on 7 March 2020 and 8 March 2020. Patient 3 (a 56-year-old male) and patient 4 (a 56-year-old female) are a married couple and residents of Wuhan, China, who traveled to Toronto, Canada, on 22 January 2020. Patient 3 first developed a cough without fever on 20 January 2020 in the city of Wuhan, where he had a normal chest X-ray on that day. He flew to Canada with persisting cough and arrived in Canada on 22 January 2020, where he became febrile. He presented to a hospital in Toronto, 23 January 2020 complaining of fever, cough and shortness of breath; a nasopharyngeal swab was positive by PCR testing for SARS-CoV-2. His chest $\mathrm{X}$-ray at that time was abnormal, and he was admitted for non-intensive-care-unit impatient care. He improved gradually with supportive care, was discharged 30 January 2020 and rapidly became asymptomatic except for a residual dry cough that persisted for a month. He had a negative nasopharyngeal swab PCR test on 19 February 2020. Patient 4 is the wife of patient 3 who traveled with her husband from Wuhan. She was never symptomatic with respiratory symptoms or fever but was tested because of her exposure. Her nasopharyngeal swab was positive for SARS-CoV-2 by PCR, on 24 January 2020; repeat testing in follow-up on 21 February 2020 was negative. PBMCs were obtained by leukapheresis from patients 3 and 4 on 10 March 2020, which was $50 \mathrm{~d}$ since symptom onset of patient 3. Samples were transferred to Vanderbilt University Medical Center in Nashville, Tennessee, on 14 March 2020.

Cell culture. Vero E6 (CRL-1586, American Type Culture (ATCC)), Vero CCL81 (CCL-81, ATCC) and HEK293T (CRL-3216 ATCC) were maintained at $37^{\circ} \mathrm{C}$ in $5 \% \mathrm{CO}_{2}$ in DMEM containing $10 \%$ (vol/vol) heat-inactivated FBS, $10 \mathrm{mM}$ HEPES $\mathrm{pH} 7.3,1 \mathrm{mM}$ sodium pyruvate, $1 \times$ non-essential amino acids, and $100 \mathrm{U} \mathrm{ml}^{-1}$ of penicillin-streptomycin. Vero-furin cells were obtained from T. Pierson (NIH) and have been described previously ${ }^{39}$. Expi293F cells (Thermo Fisher Scientific, A1452) were maintained at $37^{\circ} \mathrm{C}$ in $8 \% \mathrm{CO}_{2}$ in Expi293F Expression Medium (Thermo Fisher Scientific, A1435102). ExpiCHO cells (Thermo Fisher Scientific, A29127) were maintained at $37^{\circ} \mathrm{C}$ in $8 \% \mathrm{CO}_{2}$ in ExpiCHO Expression Medium (Thermo Fisher Scientific, A2910002). Mycoplasma testing of Expi293F and ExpiCHO cultures was performed on a monthly basis using a PCR-based mycoplasma detection kit (ATCC, 30-1012K).

Viruses. SARS-CoV-2 strain 2019 n-CoV/USA_WA1/2020 was obtained from the Centers for Disease Control and Prevention (a gift from N. Thornburg). Virus was passaged in Vero CCL81 cells and titrated by plaque assay on Vero E6 cells. All work with infectious SARS-CoV-2 was approved by the Washington University School of Medicine or UNC-Chapel Hill Institutional Biosafety Committees and conducted in approved BSL-3 facilities using appropriate powered air-purifying respirators and personal protective equipment.

Recombinant antigens and proteins. A gene encoding the ectodomain of a prefusion conformation-stabilized SARS-CoV-2 S $\left(\mathrm{S}_{2} \mathrm{P}_{\text {ecto }}\right)$ protein was synthesized and cloned into a DNA plasmid expression vector for mammalian cells. A similarly designed S protein antigen with two prolines and removal of the furin cleavage site for stabilization of the prefusion form of $\mathrm{S}$ was reported previously ${ }^{20}$. Briefly, this gene includes the ectodomain of SARS-CoV-2 (to residue 1,208), a T4 fibritin trimerization domain, an AviTag site-specific biotinylation sequence, and a carboxy-terminal $8 \times$-histidine tag. To stabilize the construct in the prefusion conformation, we included substitutions K986P and V987P and mutated the furin cleavage site at residues 682-685 from RRAR to ASVG. This recombinant spike $2 \mathrm{P}$-stabilized protein (designated here as $\mathrm{S} 2 \mathrm{P}_{\text {ecto }}$ ) was isolated by metal affinity chromatography on HisTrap Excel columns (GE Healthcare), and protein preparations were purified further by size-exclusion chromatography on a Superose 6 Increase 10/300 column (GE Healthcare). The presence of trimeric, prefusion conformation $S$ protein was verified by negative-stain electron microscopy (Extended Data Fig. 1 and Supplementary Table 2). To express the $\mathrm{S}_{\mathrm{RBD}}$ subdomain of SARS-CoV-2 S protein, residues 319-541 were cloned into a mammalian expression vector downstream of an IL-2 signal peptide and upstream of a thrombin cleavage site, an AviTag, and a $6 \times-\mathrm{His}$ tag. RBD-mFc was purchased from Sino Biological (40592-V05H). For B cell labeling and sorting, RBD-mFc and
S2P $\mathrm{P}_{\text {ecto }}$ proteins were biotinylated using the EZ-Link Sulfo-NHS-LC-Biotinylation Kit and vendor's protocol (Thermo Fisher Scientific, 21435).

\section{Electron microscopy stain grid preparation and imaging of SARS-CoV-2} S2 $P_{\text {ecto }}$ protein. For screening and imaging of negatively stained SARS-CoV-2 S2 $\mathrm{P}_{\text {ecto }}$ protein, approximately $3 \mu \mathrm{L}$ of the sample at concentrations of about 10 to $15 \mu \mathrm{g} / \mathrm{mL}$ was applied to a glow discharged grid with continuous carbon film on 400 square mesh copper EM grids (Electron Microscopy Sciences). The grids were stained with $0.75 \%$ uranyl formate $(\mathrm{UF})^{40}$. Images were recorded on a Gatan US4000 4k $\times 4 \mathrm{k}$ CCD camera using an FEI TF20 (TFS) transmission electron microscope operated at $200 \mathrm{keV}$ and control with SerialEM ${ }^{41}$. All images were taken at $\times 50,000$ magnification with a pixel size of $2.18 \AA$ pix $^{-1}$ in low-dose mode at a defocus of $1.5-1.8 \mu \mathrm{m}$. The total dose for the micrographs was $\sim 25 \mathrm{e}^{-}$per $\AA^{2}$. Image processing was performed using the cryoSPARC software package ${ }^{42}$. Images were imported, and CTF estimation was done for the particles. The images then were denoised and picked with Topaz ${ }^{43}$. The particles were extracted with a box size of 256 pixels and binned to 128 pixels. Two-dimensional class averages were performed (see Supplementary Table 2 for details).

Patient selection and target-specific memory B cells isolation. B cell responses to SARS-CoV-2 in PBMCs from a cohort of four patients with documented previous infection with the virus were analyzed for antigen specificity, and PBMCs were used for SARS-CoV-2-specific B cell enrichment. The frequency of SARS-CoV-2 $S$ protein-specific $B$ cells was identified by antigen-specific staining with either biotinylated S2P $\mathrm{P}_{\text {ecto }}$ or RBD-mFc protein. Briefly, B cells were purified magnetically (STEMCELL Technologies) and stained with anti-CD19-APC (BioLegend clone HIB19 cat. no. 982406, lot B270238, 1:10 dilution), anti-IgDFITC (BioLegend clone IA6-2, cat. no. 348206, lot B258195, 1:20 dilution), and anti-IgM-FITC (BioLegend clone MHM-88, cat. no. 314506, lot B218736, 1:20 dilution) phenotyping antibodies and biotinylated antigen. A DAPI stain was used as a viability dye to distinguish dead cells. Antigen-labeled class-switched memory B cell-antigen complexes $\left(\mathrm{CD}^{1} 9^{+} \mathrm{IgM}^{-} \mathrm{IgD}^{-} \mathrm{Ag}^{+} \mathrm{DAPI}^{-}\right)$were detected with a R-PE-labeled streptavidin conjugate (Thermo Fisher Scientific, S866, 1:500 dilution). After identification of the two patients with the highest $\mathrm{B}$ cell response against SARS-CoV-2 (patients 3 and 4), target-specific memory B cells were isolated by flow-cytometric sorting using an SH800 cell sorter (Sony) from pooled PBMCs of these two patients, after labeling of B cells with either biotinylated $\mathrm{S} 2 \mathrm{P}_{\text {ecto }}$ or RBD-mFc proteins. Flow-cytometric data were analyzed with the SH800 software and FlowJo version 10 (Tree Star).

Overall, from $>4 \times 10^{8}$ PBMCs, 2,126 RBD-mFc-reactive and 5,544 $\mathrm{S} 2 \mathrm{P}_{\text {ecto }}$-reactive B cells were sorted and subjected to further analysis. Several methods were implemented for the preparation of sorted B cells for sequencing. Approximately 4,500 sorted cells were subjected to direct sequencing immediately after flow cytometric sorting. The remaining cells were expanded in culture for $8 \mathrm{~d}$ in the presence of irradiated 3T3 feeder cells that were engineered to express human CD40L, IL-21 and BAFF, as described previously ${ }^{22}$. The expanded lymphoblastoid cell lines (LCLs) secreted high levels of S-protein-specific antibodies, as confirmed by ELISA, to detect antigen-specific human antibodies in culture supernatants. Approximately 40,000 expanded LCLs were sequenced using the Chromium sequencing method (10x Genomics).

Microfluidic device selection of single antigen-specific B cells. Activated memory B cells were screened using Berkeley Lights' Beacon optofluidic system. Purified B cell samples were imported automatically onto OptoSelect $11 \mathrm{k}$ chips in a novel plasmablast survival medium that promotes antibody secretion and preserves cell viability ${ }^{44}$. Single-cell penning was then performed using OEP technology, in which light is used to transfer B cells into individual nanoliter-volume chambers (NanoPens). Using this light-based manipulation, thousands of LCLs were transferred into pens across multiple chips in each workflow. We performed an on-chip, fluorescence-based assay to identify antibodies that bound SARS-CoV-2 S2P $\mathrm{P}_{\text {ecto }}$ or RBD-mFc protein. We prepared 6- to $8-\mu \mathrm{m}$ and 10 - to $14-\mu \mathrm{m} \mathrm{RBD}-\mathrm{mFc}$-conjugated beads by coupling biotinylated $\mathrm{RBD}-\mathrm{mFc}$ protein to streptavidin-coated polystyrene particles (Spherotech). We prepared 6- to 8- $\mu \mathrm{m} \mathrm{S2} \mathrm{P}_{\text {ecto }}$ protein-conjugated beads by coupling full-length $\mathrm{S}_{2} \mathrm{P}_{\text {ecto }}$ protein to streptavidin-coated polystyrene particles. Assays consisted of mixing beads conjugated with the RBD- $\mathrm{mFc}$ or $\mathrm{S} 2 \mathrm{P}_{\text {ecto }}$ proteins with Alexa Fluor (AF)-labeled anti-human secondary antibodies (Thermo Fisher Scientific cat. no. A-21090) at a 1:100 dilution and importing this assay mixture into OptoSelect $11 \mathrm{k}$ chips. Antigen-specific antibodies bound the antigen-conjugated beads, which then sequestered the fluorescent secondary antibody. Cells secreting antigen-specific antibodies were identified by locating the NanoPens immediately adjacent to the fluorescent beads. We also performed an on-chip assay to select antibodies that blocked the interaction of hACE2 and the RBD of SARS-CoV-2. The blocking assay was performed by first co-incubating LCLs and 10- to $14-\mu \mathrm{m}$ RBD-mFc-conjugated streptavidin-coated beads (Spherotech) in the NanoPen chambers to allow for secreted antibodies to saturate the antigen. Then, a mixture of recombinant hACE2 with a FLAG tag (Sigma-Aldrich cat. no. SAE0064), a rat anti-FLAG AF 647 antibody at a 1:50 dilution (BioLegend clone L5, cat. no. 637315, Lot B265929) and an anti-human IgG AF 568 antibody at a 1:100 dilution (Thermo 
Fisher Scientific cat. no. A-21090) was perfused through the OptoSelect 11k chip and allowed to diffuse into the NanoPen chambers. RBD-binding antibodies were identified by locating pens with RBD-mFc-conjugated beads that were fluorescent when imaged using the Beacon TRED filter cube. Simultaneously, hACE2 binding to the RBD-coated beads was detected using a Cy5 filter cube. NanoPen chambers containing $\mathrm{RBD}-\mathrm{mFc}$-conjugated beads with fluorescence in both filter cubes were classified as containing B cells secreting anti-SARS-CoV-2 antibodies that bound RBD but that did not demonstrate hACE2-blocking activity. NanoPen chambers that contained RBD-mFc-conjugated beads that were fluorescent in the TRED channel but non-fluorescent in the Cy5 channel contained secreted antibodies that had both bound RBD and blocked hACE2-RBD interaction. Antigen-specific cells of interest were exported from specific NanoPen chambers to individual wells of 96-well reverse transcription-PCR plates containing lysis buffer.

\section{Sequencing and cloning of single antigen-specific B cells. After export from} the Beacon instrument, antibody heavy- and light-chain sequences for B cells secreting RBD-mFc- or $\mathrm{S} 2 \mathrm{P}_{\text {ecto }}$-binding antibodies were amplified and recovered using components of the Opto Plasma B Discovery cDNA Synthesis Kit (Berkeley Lights). Antibody heavy- and light-chain sequences were amplified through a 5 ' RACE approach using the kit's included 'BCR Primer 2' forward primer and isotype-specific reverse primers. The 5'-RACE-amplified cDNA was sequenced using the Pacific Biosciences Sequel platform using the SMRTbell Barcoded Adapt Complete Prep-96 kit (Pacific Biosciences) and a 6-h movie time. In a redundant sequencing approach, heavy- and light-chain sequences were amplified using a cocktail of custom V- and J-gene-specific primers (similar to previously described human Ig gene-specific primers ${ }^{45}$ ) from the original 5'-RACE-amplified cDNA while the products of the gene-specific amplification were sent for Sanger sequencing (GENEWIZ). The sequences generated by these two approaches were analyzed using our Python-based antibody variable-gene analysis tool (PyIR; https://github.com/crowelab/PyIR $)^{46}$ to identify which $V$ and $J$ genes most closely matched the nucleotide sequence. Heavy- and light-chain sequences were then amplified from the original cDNA using cherry-picked $V$-and $J$-gene-specific primers most closely corresponding to the $V$ and $J$ gene calls made by PyIR. These primers include adapter sequences which allow Gibson-based cloning into a monocistronic IgG1 expression vector (pMCis_G1). Similar to a vector described below, this vector contains an enhanced 2A sequence and GSG linker that allows simultaneous expression of $\mathrm{mAb}$ heavy and light chain genes from a single construct upon transfection ${ }^{47}$. The pMCis_G1 vector was digested using the New England BioLabs restriction enzyme FspI, and the amplified paired heavy- and light-chain sequences were cloned through Gibson assembly using NEBuilder HiFi DNA Assembly Master Mix. After recovered sequences were cloned into pMCis_ $\mathrm{G} 1$ expression constructs, recombinant antibodies were expressed in $\mathrm{CHO}$ cells and were purified by affinity chromatography as detailed below. Antigen-binding activity was confirmed using plate-based ELISA.

Generation of antibody variable-gene libraries from single B cells. As an alternative approach, we also used a second major approach for isolation of SARS-CoV-2-reactive antibodies. In some experiments, the Chromium Single Cell V(D)J workflow with B-cell-only enrichment option was used for generating linked heavy-chain and light-chain antibody profiling libraries. Approximately 2,866 directly sorted S2 $\mathrm{P}_{\text {ecto }}$ or 1,626 RBD- $\mathrm{mFc}$ protein-specific B cells were split evenly into 2 replicates each and separately added to $50 \mu$ l of RT Reagent Mix, $5.9 \mu \mathrm{l}$ of Poly-dt RT Primer, $2.4 \mu \mathrm{l}$ of Additive A and $10 \mu \mathrm{l}$ of RT Enzyme Mix B to complete the Reaction Mix as per the vendor's protocol, which then was loaded directly onto a Chromium chip (10x Genomics). Similarly, for the remaining sorted cells that were expanded in bulk, approximately 40,000 cells from 2 separate sorting approaches were split evenly across 4 reactions and processed separately as described above, before loading onto a Chromium chip. The libraries were prepared following the User Guide for Chromium Single Cell V(D)J Reagents kits (CG000086_REV K).

Next-generation DNA sequence analysis of antibody variable genes. Chromium Single Cell V(D)J B-Cell-enriched libraries were quantified, normalized and sequenced according to the User Guide for Chromium Single Cell V(D)J Reagents kits (CG000086_REV C). The two enriched libraries from direct flow cytometric cell sorting were sequenced on a NovaSeq sequencer (Illumina) with a NovaSeq 6000 S1 Reagent Kit (300 cycles) (Illumina). The four enriched libraries from bulk expansion were sequenced on a NovaSeq sequencer with a NovaSeq 6000 S4 Reagent Kit (300 cycles) (Illumina). All enriched V(D)J libraries were targeted for sequencing depth of at least 5,000 raw read pairs per cell.

Bioinformatics analysis of single-cell sequencing data. Following sequencing, all samples were demultiplexed and processed through the 10x Genomics Cell Ranger software (version 2.1.1). The down-selection to identify lead candidates for expression was carried out in two phases. In the first phase, all paired antibody heavy- and light-chain variable-gene cDNA nucleotide sequences obtained that contained a single heavy- and light-chain sequence were processed using PyIR. We considered heavy- and light-chain-encoding gene pairs productive and retained them for additional downstream processing if they met the following criteria: (1) did not contain a stop codon, (2) encoded an intact CDR3 and (3) contained an in-frame junctional region. The second phase of processing eliminated redundant sequences (those with identical amino acid sequences). Any antibody variant that was designated as an IgM isotype (based on the sequence and assignment using the 10x Genomics Cell Ranger V(D)J software, version 2.1.1) was removed from consideration (while IgG and IgA isotype antibodies were retained). The identities of antibody variable-gene segments, CDRs and mutations from inferred germline gene segments were determined by using PyIR.

\section{Antibody gene synthesis. Sequences of selected mAbs were synthesized using} a rapid high-throughput cDNA synthesis platform (Twist Bioscience) and subsequently cloned into an IgG1 monocistronic expression vector (designated as pTwist-mCis_G1) for mammalian cell culture mAb secretion. This vector contains an enhanced 2A sequence and GSG linker that allows simultaneous expression of $\mathrm{mAb}$ heavy- and light-chain genes from a single construct upon transfection ${ }^{47}$.

\section{MAb production and purification. For high-throughput production of} recombinant $\mathrm{mAbs}$, we adopted approaches designated as 'micro-scale' or 'midi-scale'. For 'micro-scale' mAbs expression, we performed micro-scale transfection ( $\sim 1 \mathrm{ml}$ per antibody) of CHO cell cultures using the Gibco ExpiCHO Expression System and a protocol for deep 96-well blocks (Thermo Fisher Scientific), as previously described ${ }^{19}$. Briefly, synthesized antibody-encoding lyophilized DNA was reconstituted in OptiPro serum-free medium (OptiPro SFM) and used for transfection of ExpiCHO cell cultures into 96-deep-well blocks. For high-throughput micro-scale $\mathrm{mAb}$ purification, clarified culture supernatants were incubated with MabSelect SuRe resin (Cytiva), washed with PBS, eluted, buffer-exchanged into PBS using Zeba Spin Desalting Plates (Thermo Fisher Scientific) and stored at $4^{\circ} \mathrm{C}$ until use. For 'midi-scale' mAbs expression, we performed transfection $(\sim 15 \mathrm{ml}$ per antibody) of $\mathrm{CHO}$ cell cultures using the Gibco ExpiCHO Expression System and protocol for $50 \mathrm{ml}$ mini bioreactor tubes (Corning), as described by the vendor. For high-throughput midi-scale $\mathrm{mAb}$ purification, culture supernatants were purified using HiTrap MabSelect SuRe (Cytiva) on a 24-column parallel protein chromatography system (Protein BioSolutions). Purified mAbs were buffer-exchanged into PBS, concentrated using Amicon Ultra-4 $50 \mathrm{KDa}$ Centrifugal Filter Units (Millipore Sigma) and stored at $4^{\circ} \mathrm{C}$ until use.

ELISA binding screening assays. Wells of 96-well microtiter plates were coated with purified recombinant SARS-CoV-2 S protein, SARS-CoV-2 $\mathrm{S}_{\mathrm{RBD}}$ protein, SARS-CoV-2 $\mathrm{S}_{\mathrm{NTD}}$ (kindly provided by P. McTamney, K. Ren and A. Barnes, Astra Zeneca) or SARS-CoV S protein (kindly provided by S. Bangaru and A. Ward, Scripps Research Institute) at $4{ }^{\circ} \mathrm{C}$ overnight. Plates were blocked with $2 \%$ non-fat dry milk and $2 \%$ normal goat serum in DPBS containing $0.05 \%$ Tween-20 (DPBS-T) for $1 \mathrm{~h}$. For mAb screening assays, Supernatants from $\mathrm{CHO}$ cell culture or purified mAbs were diluted 1:20 in blocking buffer, added to the wells and incubated for $1 \mathrm{~h}$ at ambient temperature. The bound antibodies were detected using goat anti-human IgG conjugated with horseradish peroxidase (Southern Biotech) and TMB substrate (Thermo Fisher Scientific). Color development was monitored, $1 \mathrm{~N}$ hydrochloric acid was added to stop the reaction and the absorbance was measured at $450 \mathrm{~nm}$ using a spectrophotometer (Biotek).

RTCA. To screen for neutralizing activity in the panel of recombinantly expressed mAbs, we used a high-throughput and quantitative RTCA assay and xCelligence RTCA HT Analyzer (ACEA Biosciences) that assesses kinetic changes in cell physiology, including virus-induced cytopathic effect (CPE). Twenty $\mu$ l of cell culture medium (DMEM supplemented with $2 \%$ FBS) was added to each well of a 384-well E-plate using a ViaFlo384 liquid handler (Integra Biosciences) to obtain background reading. Six thousand $(6,000)$ Vero-furin cells in $20 \mu$ l of cell culture medium were seeded per well, and the plate was placed on the analyzer. Sensograms were visualized using RTCA HT software version 1.0.1 (ACEA Biosciences). For a screening neutralization assay, equal amounts of virus were mixed with micro-scale purified antibodies in a total volume of $40 \mu$ using DMEM supplemented with $2 \% \mathrm{FBS}$ as a diluent and incubated for $1 \mathrm{~h}$ at $37^{\circ} \mathrm{C}$ in $5 \% \mathrm{CO}_{2}$. At $\sim 17-20 \mathrm{~h}$ after seeding the cells, the virus $-\mathrm{mAb}$ mixtures were added to the cells in 384-well E-plates. Wells containing virus only (in the absence of $\mathrm{mAb}$ ) and wells containing only Vero cells in medium were included as controls. Plates were measured every $8-12 \mathrm{~h}$ for $48-72 \mathrm{~h}$ to assess virus neutralization. Micro-scale antibodies were assessed in four 5-fold dilutions (starting from a 1:20 sample dilution), and their concentrations were not normalized. In some experiments, mAbs were tested in triplicate using a single (1:20) dilution. Neutralization was calculated as the percent of maximal cell index in control wells without virus minus cell index in control (virus-only) wells that exhibited maximal CPE at $40-48 \mathrm{~h}$ after applying virus-antibody mixture to the cells. A mAb was classified as fully neutralizing if it completely inhibited SARS-CoV-2-induced CPE at the highest tested concentration, while a mAb was classified as partially neutralizing if it delayed but did not fully prevent CPE at the highest tested concentration. Representative sensograms for fully neutralizing and partially neutralizing mAbs are shown in Extended Data Fig. 4. For mAb potency ranking experiments, individual mAbs identified as fully neutralizing from the screening study were assessed by FRNT. 
Sequence analysis of antigen-reactive $\mathbf{m A b}$ sequences. Sequences of the 389 $\mathrm{mAbs}$ isolated by different approaches were combined and run through PyIR to identity the $\mathrm{V}$ genes, J genes, CDR3 lengths and percent identity to germline, and sequence within the FR1-FR4 region for both heavy and light chains. Sequences were then deduplicated on the nucleotide sequences identified in the FR1-FR4 region. Among the $389 \mathrm{mAbs}$, there were 324 unique nucleotide sequences (321 unique amino acid sequences) that were analyzed for $V / D / J$ gene usage, CDR3 length and somatic mutation. First, the number of sequences with corresponding $V$ and $J$ genes were counted. The $V / J$ frequency counts were then transformed into a $Z$-score by first subtracting away the average frequency then normalizing by the s.d. of the total set of antigen-reactive mAbs. The $Z$-score was then plotted as a heatmap using python seaborn library. The amino acid length of each CDR3 was counted. The distribution of CDR3 amino acid lengths were then plotted as histograms and fitted using kernel density estimation for the curves using python seaborn library. The number of mutations for each $\mathrm{mAb}$ relative to the inferred germline variable-gene sequence was counted for each chain. This number was then transformed into a percentage value. These values were then plotted as a categorical distribution plot as a violin plot using the python seaborn. catplot library.

FRNT. Serial dilutions of serum or plasma were incubated with $10^{2} \mathrm{FFU}$ of SARS-CoV- 2 for $1 \mathrm{~h}$ at $37^{\circ} \mathrm{C}$. The serum-virus or plasma-virus mixtures were then added to Vero E6 cell monolayers in 96-well plates for $1 \mathrm{~h}$ at $37^{\circ} \mathrm{C}$. Subsequently, cells were overlaid with $1 \%$ (wt/vol) methylcellulose in Minimum Essential Medium (MEM) supplemented to contain $2 \%$ heat-inactivated FBS. Plates were fixed $30 \mathrm{~h}$ later by removing overlays and were fixed with $4 \%$ PFA in PBS for $20 \mathrm{~min}$ at room temperature. The plates were incubated sequentially with $1 \mu \mathrm{g} \mathrm{ml}^{-1}$ rCR3022 anti-S antibody ${ }^{5}$ and a goat anti-human IgG ( $\gamma$-chain specific)-peroxidase antibody (Sigma-Aldrich, cat. no. A6029) in PBS supplemented with $0.1 \%$ (wt/vol) saponin (Sigma) and $0.1 \%$ BSA. SARS-CoV-2-infected cell foci were visualized using TrueBlue peroxidase substrate (KPL) and quantitated on an ImmunoSpot 5.0.37 Macro Analyzer (Cellular Technologies).

SARS-CoV or SARS-CoV-2 neutralization assays using SARS-CoV or SARS-CoV-2 luciferase reporter viruses. Full-length viruses expressing luciferase were designed and recovered via reverse genetics as described previously ${ }^{48,49}$. Viruses were titered in Vero E6 cell culture monolayers to obtain a relative light units (RLU) signal of at least $20 \times$ the cell-only control background. Vero E6 cells were plated at 20,000 cells per well the day prior in clear-bottom black-walled 96-well plates (Corning no. 3904). Neutralizing antibodies were diluted serially by fourfold for up to eight dilutions. SARS-Urbani NanoLuc or SARS-CoV-2 NanoLuc virus were mixed with serially diluted antibodies. Antibody-virus complexes were incubated at $37^{\circ} \mathrm{C}$ in $5 \% \mathrm{CO}_{2}$ for $1 \mathrm{~h}$. Following incubation, growth medium was removed, and virus-antibody dilution complexes were added to the cells in duplicate. Virus-only and cell-only controls were included in each neutralization-assay plate. Following infection, plates were incubated at $37^{\circ} \mathrm{C}$ in $5 \% \mathrm{CO}_{2}$ for $48 \mathrm{~h}$. After the 48 -h incubation, cells were lysed and luciferase activity was measured using the Nano-Glo Luciferase Assay System (Promega), according to the manufacturer's specifications.

Neutralization assays using a recombinant VSV-SARS-CoV-2 virus. Neutralization assays using replication-competent VSV expressing enhanced green fluorescent protein (eGFP) and the SARS-CoV-2 S protein with a C-terminal deletion were performed, as previously described ${ }^{25}$. Briefly, indicated dilutions of $\mathrm{mAbs}$ were incubated with VSV-SARS-CoV-2 for $1 \mathrm{~h}$ at $37^{\circ} \mathrm{C}$. Antibody-virus complexes then were added to Vero E6 cells in 96-well plates and incubated at $37^{\circ} \mathrm{C}$ for $7.5 \mathrm{~h}$. Cells were fixed at room temperature in $2 \%$ formaldehyde containing $10 \mu \mathrm{g} \mathrm{ml}^{-1}$ Hoechst 33342 nuclear stain for $45 \mathrm{~min}$. Fixative was replaced with PBS prior to imaging. Images were acquired using an InCell 2000 Analyzer automated microscope (GE Healthcare) in both the DAPI and FITC channels to visualize nuclei and infected cells $(\times 4$ objective, 4 fields per well). Images were analyzed using the Multi Target Analysis Module of the InCell Analyzer 1000 Workstation Software (GE Healthcare). GFP-positive cells were identified using the top hat segmentation method and counted within the InCell Workstation software. Neutralization assay data are summarized in Extended Data Fig. 6.

High-throughput mAb quantification. High-throughput quantification of micro-scale-produced mAbs was performed from CHO culture supernatants or micro-scale purified mAbs in a 96-well plate format using the Cy-Clone Plus Kit and an iQue Plus Screener flow cytometer (IntelliCyt Corp), according to the vendor's protocol. Purified mAbs were assessed at a single dilution (1:10 final, using $2 \mu$ l of purified $\mathrm{mAb}$ per reaction), and a control human IgG solution with known concentration was used to generate a calibration curve. Data were analyzed using ForeCyt software version 6.2 (IntelliCyt Corp).

Quantification and statistical analysis. The descriptive statistics mean \pm SEM or mean \pm SD were determined for continuous variables as noted. Technical and biological replicates are described in the figure legends. Statistical analyses were performed using Prism v8.0 (GraphPad).
Reporting Summary. Further information on research design is available in the Nature Research Reporting Summary linked to this article.

\section{Data availability}

The main data supporting the results in this study are available within the paper and Supplementary Information. The ImMunoGeneTics database is available from http://www.imgt.org/. The analysis pipeline PyIR (https://github.com/crowelab/ PyIR) and the specific scripts used for sequence analysis (https://github.com/ crowelab/cov2-panel-scripts) are available. Structures deposited by other groups for the full-length spike trimer (6VYB) and the RBD-hACE2 complex (6M0J) that were used for visualization in this paper are publicly available (www.rcsb. org). Sequences for mAbs described in this study have been deposited at GenBank and are available under the following accession codes: MT665032-MT665070, MT665419-MT665457, MT665071-MT665418 and MT665458-MT665805. Datasets are available from the corresponding authors upon reasonable request.

\section{References}

39. Mukherjee, S. et al. Enhancing dengue virus maturation using a stable furin over-expressing cell line. Virology 497, 33-40 (2016).

40. Ohi, M., Li, Y., Cheng, Y. \& Walz, T. Negative staining and image classification-powerful tools in modern electron microscopy. Biol. Proced. Online 6, 23-34 (2004).

41. Mastronarde, D. N. Automated electron microscope tomography using robust prediction of specimen movements. J. Struct. Biol. 152, 36-51 (2005).

42. Punjani, A., Rubinstein, J. L., Fleet, D. J. \& Brubaker, M. A. cryoSPARC: algorithms for rapid unsupervised cryo-EM structure determination. Nat. Methods 14, 290-296 (2017).

43. Bepler, T., Noble, A. J. \& Berger, B. Topaz-Denoise: general deep denoising models for cryoEM. Preprint at https://doi.org/10.1101/838920 (2019).

44. Nguyen, D. C. et al. Factors of the bone marrow microniche that support human plasma cell survival and immunoglobulin secretion. Nat. Commun. 9 , 3698 (2018).

45. Guthmiller, J. J., Dugan, H. L., Neu, K. E., Lan, L. Y. \& Wilson, P. C. An efficient method to generate monoclonal antibodies from human B cells. Methods Mol. Biol. 1904, 109-145 (2019).

46. Soto C. F. J. et al. PyIR: A scalable wrapper for processing billions of immunoglobulin and $\mathrm{T}$ cell receptor sequences using IgBLAST. BMC Bioinformatics (in the press).

47. Chng, J. et al. Cleavage efficient $2 \mathrm{~A}$ peptides for high level monoclonal antibody expression in CHO cells. MAbs 7, 403-412 (2015).

48. Scobey, T. et al. Reverse genetics with a full-length infectious cDNA of the Middle East respiratory syndrome coronavirus. Proc. Natl Acad. Sci. USA 110, 16157-16162 (2013).

49. Yount, B. et al. Reverse genetics with a full-length infectious cDNA of severe acute respiratory syndrome coronavirus. Proc. Natl Acad. Sci. USA 100, 12995-13000 (2003).

\section{Acknowledgements}

We thank M. Mayo and N. S. Galeano for coordination of human studies, D. O'Connor, N. Safdar, G. Baird, J. Shendure and S. Mubareka for helpful advice on human patients, A. Jones and the staff of the Vanderbilt VANTAGE core laboratory for expedited sequencing, R. Trosseth for assistance with data management and analysis, R. Bombardi and C. Soto for technical consultation on genomics approaches, A. Kim for production of a recombinant form of the mAb CR3022, C. Swearingen and the staff of Fedex Express Specialty Services for expedited transport services, V. Pai and K. Breinlinger of Berkeley Lights, and K. Louder and scientists at Twist Bioscience, B. Fritz at 10x Genomics and representatives at ACEA Biosciences for providing resources, outstanding expedited services and expert applications support. We thank A. Ward, S. Bangaru, P. McTamney, $\mathrm{K}$. Ren and A. Barnes for protein reagents. This study was supported by Defense Advanced Research Projects Agency (DARPA) grant HR0011-18-2-0001, NIH contracts 75N93019C00074 and 75N93019C00062 and the Dolly Parton COVID-19 Research Fund at Vanderbilt. This work was supported by NIH grant 1S10RR028106-01A1 for the Next Generation Nucleic Acid Sequencer, housed in Vanderbilt Technologies for Advanced Genomics (VANTAGE) and supported by the National Center for Research Resources, grant UL1 RR024975-01, and is now at the National Center for Advancing Translational Sciences, grant 2 UL1 TR000445-06. S.J.Z. was supported by NIH T32 AI095202. J.B.C. is supported by a Helen Hay Whitney Foundation postdoctoral fellowship. D.R.M. was supported by NIH T32 AI007151 and a Burroughs Wellcome Fund Postdoctoral Enrichment Program Award. J.E.C. is the recipient of the 2019 Future Insight Prize from Merck KGaA, which supported this research with a research grant. The content is solely the responsibility of the authors and does not necessarily represent the official views of the US government or the other sponsors.

\section{Author contributions}

Conceived of the project: S.J.Z., P.G., R.H.C., L.B.T., M.S.D., J.E.C.; Obtained funding: J.E.C. and M.S.D. Obtained human samples: M.O., H.Y.C., J.E.C.; Performed laboratory experiments: S.J.Z., P.G., R.E.C., J.B.C., J.X.R., A.T., R.S.N., R.E.S., N.S., E.B., J.E.D., 
K.W.M., S.S., D.R.M, P.W.R., L.-M.B.; Performed computational work: E.C.C., T.J., S.D., L.M.; Supervised research: S.P.J.W. M.S.D., L.B.T., R.S.B., R.H.C., J.E.C.; Provided critical reagents: J.E.D., K.W.M., F.E.-H.L., D.C.N., I.S., R.S.B.; Wrote the first draft of the paper: S.J.Z., P.G., R.H.C., J.E.C. All authors reviewed and approved the final manuscript.

\section{Competing interests}

R.S.B. has served as a consultant for Takeda and Sanofi Pasteur on issues related to vaccines. M.S.D. is a consultant for Inbios, Vir Biotechnology, NGM Biopharmaceuticals and Eli Lilly, is on the scientific advisory board of Moderna and is a recipient of unrelated research grants from Moderna and Emergent BioSolutions. H.Y.C. has served as a consultant for Merck and GlaxoSmithKline and has received research funding from Sanofi Pasteur and research support from Cepheid, Genentech and Ellume. J.E.C. has served as a consultant for Sanofi and is on the scientific advisory boards of CompuVax and Meissa Vaccines, is a recipient of previous unrelated research grants from Moderna and Sanofi and is founder of IDBiologics. Vanderbilt University has applied for patents concerning SARS-CoV-2 antibodies that are related to this work. Emory University has applied for a patent concerning the plasmablast survival medium. S.P.J.W. and P.W.R. have filed a disclosure with Washington University for the recombinant VSV. J.E.D. and K.W.M. are employees of Berkeley Lights. All other authors declared no competing interests.

\section{Additional information}

Extended data is available for this paper at https://doi.org/10.1038/s41591-020-0998-x. Supplementary information is available for this paper at https://doi.org/10.1038/ s41591-020-0998-x.

Correspondence and requests for materials should be addressed to R.H.C. or J.E.C.

Peer review information Joao Monteiro was the primary editor on this article and managed its editorial process and peer review in collaboration with the rest of the editorial team.

Reprints and permissions information is available at www.nature.com/reprints. 
a

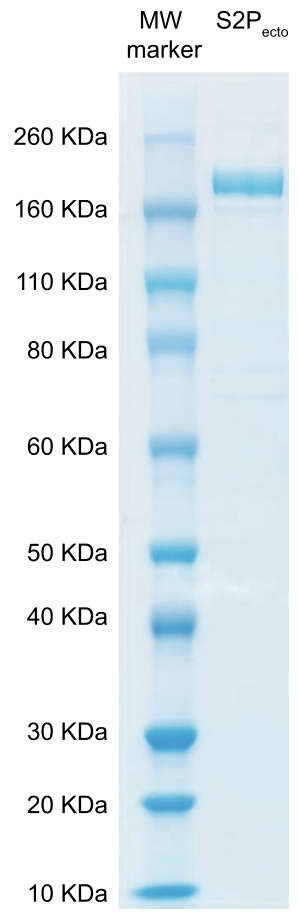

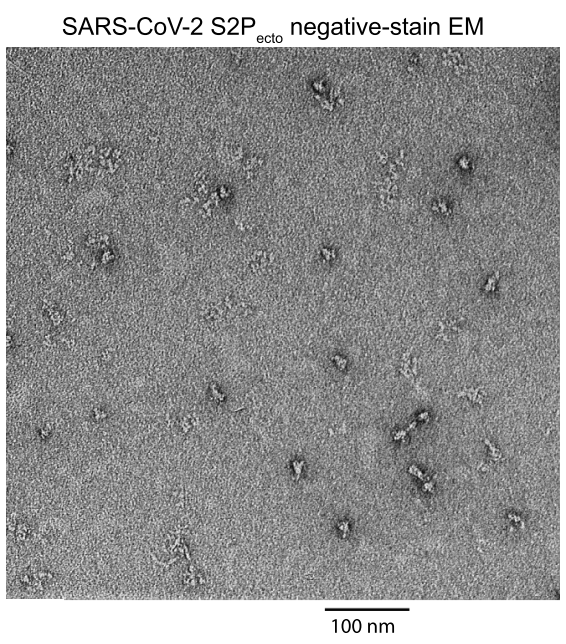

C

SARS-CoV-2 $\mathrm{S}_{2} \mathrm{P}_{\text {ecto }} 2 \mathrm{D}$ class-averages
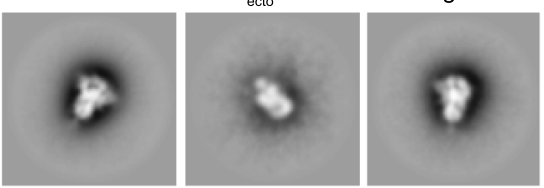

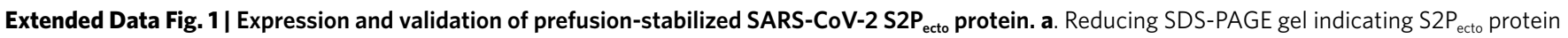
migrating at approximately $180 \mathrm{KDa}$. One representative protein preparation is shown. $\mathbf{b}$. Representative micrograph of negative-stain electron microscopy

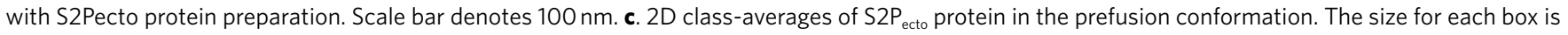
128 pixels. Detailed information on image collection is available in Supplementary Table 2. 


\section{NATURE MEDICINE}

LETTERS
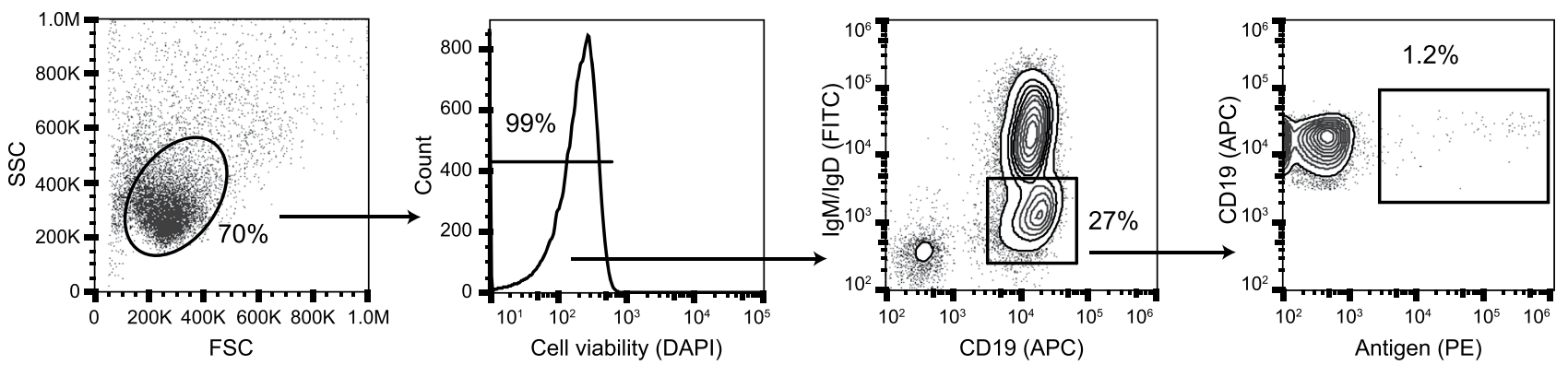

Extended Data Fig. 2 | Representative gating strategy for antigen-specific cell sorting. Representative gating strategy for profiling of antigen-specific B cell frequency for donors. Subject 4 is shown, and phenotypic markers are shown on plot axes. Arrows indicate cell populations derived from gates. 
a

b

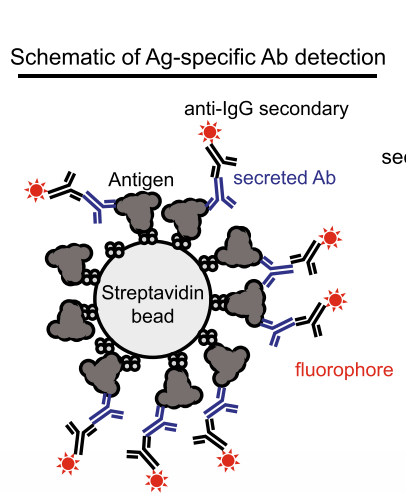

b beads

in channel

$\therefore \circ: 0 \%:$

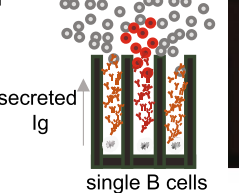

Binding assay with $\mathrm{S}_{2} \mathrm{P}_{\text {ecto }}$-coated beads

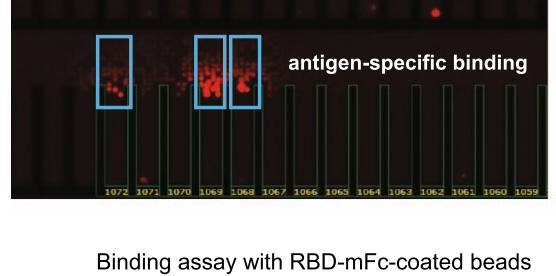

in pens
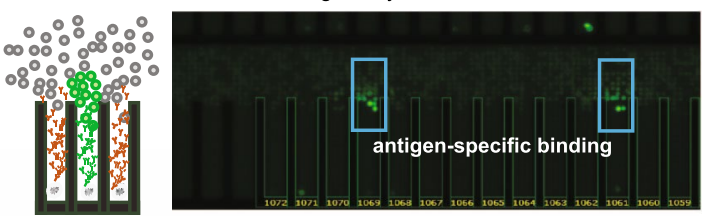

C

RBD-mFc-reactive B cells

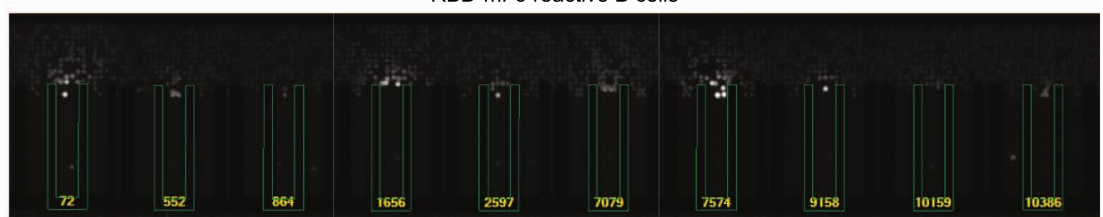

- in-channel

Schematic of hACE2 non-blocking mAb

binding assay
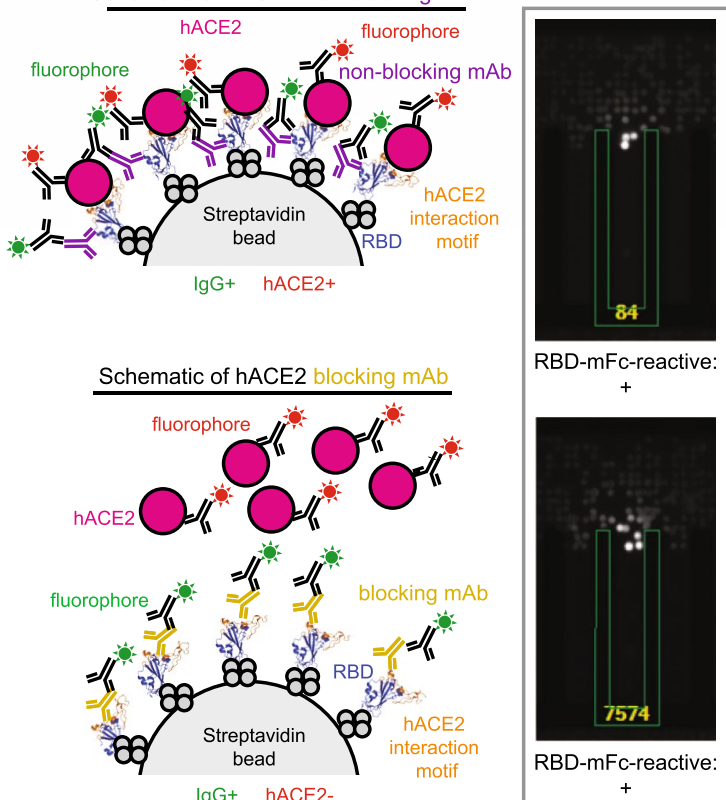

RBD-mFc-reactive:

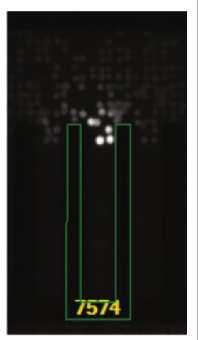

RBD-mFc-reactive:

$+$

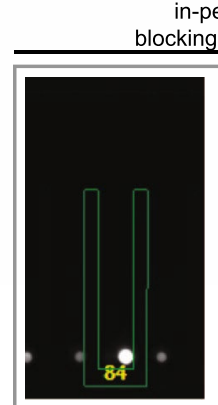

RBD binding:

$+$

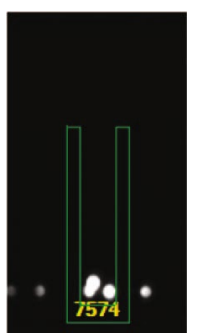

RBD binding:

-pen

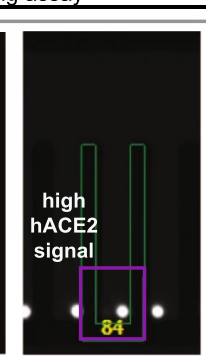

hACE2 binding:

$+$

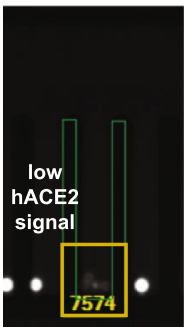

hACE2 binding:

Extended Data Fig. 3 | Functional assays from single antigen-reactive B cells. a. Biotinylated antigen (dark grey) was coupled to a streptavidin-conjugated polystyrene bead (light grey). Antibodies (blue) are secreted by single B cells loaded into individual NanoPens on the Berkeley Lights Beacon optofluidic device. Antibody binding to antigen was detected with a fluorescent anti-human IgG secondary Ab (black). b. Left: Schematic of fluorescing beads in the channel above a pen containing an individual B cell indicates antigen-specific reactivity. Top right: False-color still image of positive wells with B cells

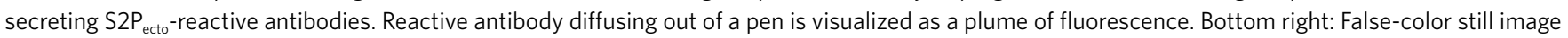
of positive wells with $B$ cells secreting RBD-mFc-reactive antibodies. $c$. Representative images of RBD-mFc reactive $B$ cells from a single-B-cell secretion assay. $\mathbf{d}$. Identification of $\mathrm{mAbs}$ with hACE2-blocking activity using single-cell functional screening. Left: Schematic illustrating detection of secreted $A b$ and hACE2 binding on an RBD-mFc-coated streptavidin bead. Ab binding was detected in one fluorescent channel, while hACE2 binding was detected in another fluorescent channel. The top panel illustrates an RBD-binding, non-blocking mAb, where the bead is positive for both Ab and hACE2 signals, while the bottom panel illustrates an RBD-binding mAb that competes with hACE2 for binding, where the bead is positive for only Ab signal. Right: Representative images of a B cell secreting non-blocking Abs (top) and a B cell secreting hACE2-blocking mAbs (bottom). Streptavidin beads are loaded into the same pens as B cells. The fluorescence of the streptavidin beads in the same pen as the B cell secreting hACE2-blocking Abs is reduced relative to adjacent wells, indicating hACE2-blocking activity. 
$\mathbf{a}$

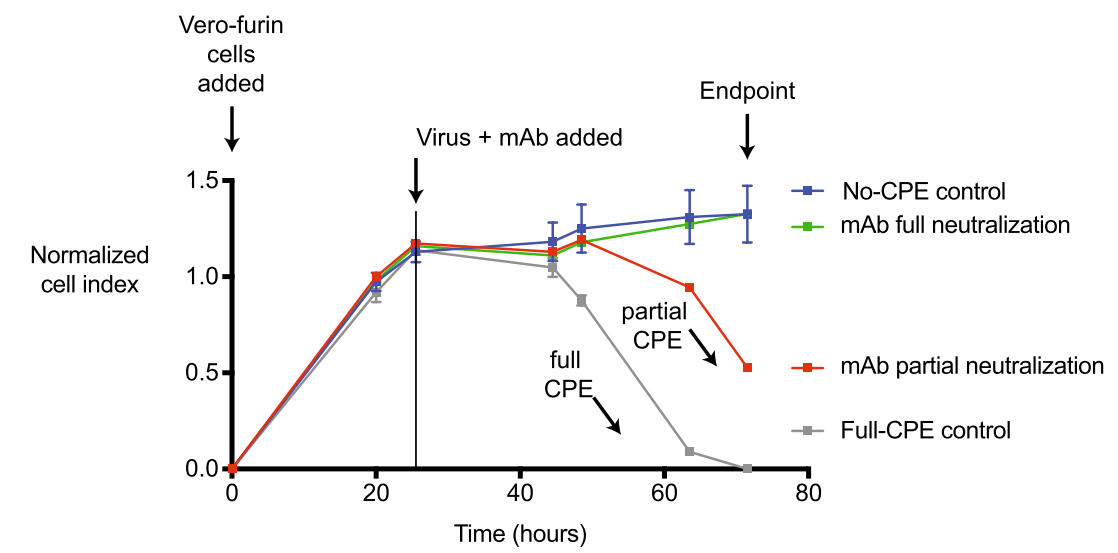

b

SARS-CoV-2 CPE measurement over time, in 384-well plate assay

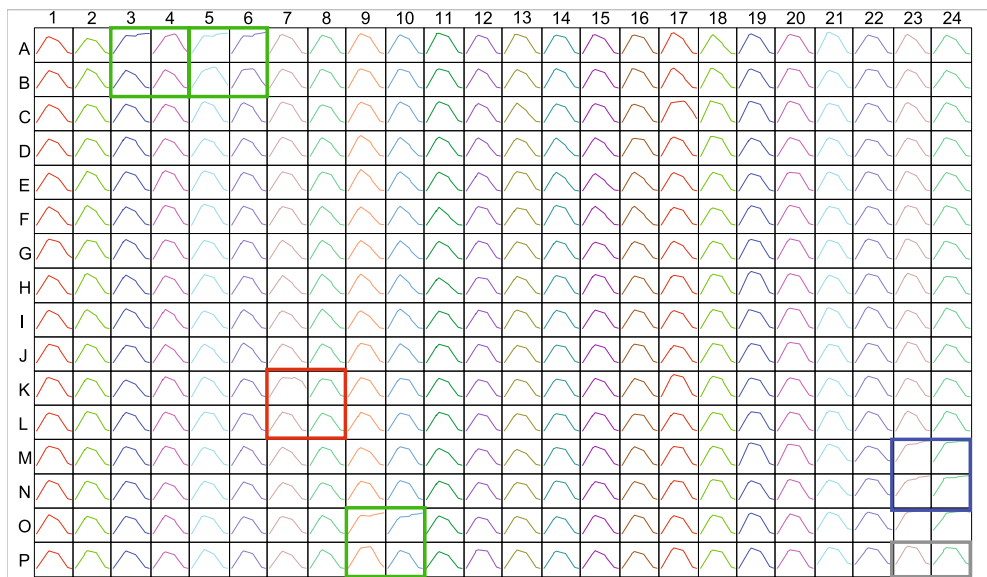

MAb

fold-dilution schema

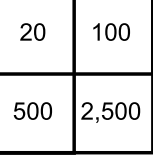

MAb neutralizing activity

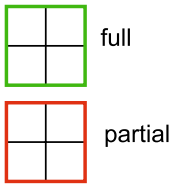

Control wells

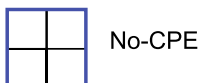

Full-CPE

Extended Data Fig. 4 | Real-time cell analysis assay to screen for neutralization activity. a. Curves for fully neutralizing mAb (green) and partially neutralizing $\mathrm{mAb}$ (red) by monitoring of CPE in Vero-furin cells that were inoculated with SARS-CoV-2 and pre-incubated with the respective $m A b$. Uninfected cells (blue) and infected cells without antibody addition (grey) served as controls for intact monolayer and full CPE, respectively. Data represent a single well measurement for each $\mathrm{mAb}$ at the highest tested concentration, mean $\pm \mathrm{SD}$ values of technical duplicates for the positive $\mathrm{CPE}$ control, and mean \pm SD values of technical quadruplicates for the no-CPE controls. b. Example sensograms from individual wells of 384-well E-plate analysis showing rapid identification of SARS-CoV-2 neutralizing mAbs. Neutralization was assessed using micro-scale purified mAbs and each mAb was tested in four 5-fold dilutions as indicated. Plates were measured every 8-12 hrs for a total of 72 hrs as in (a). 

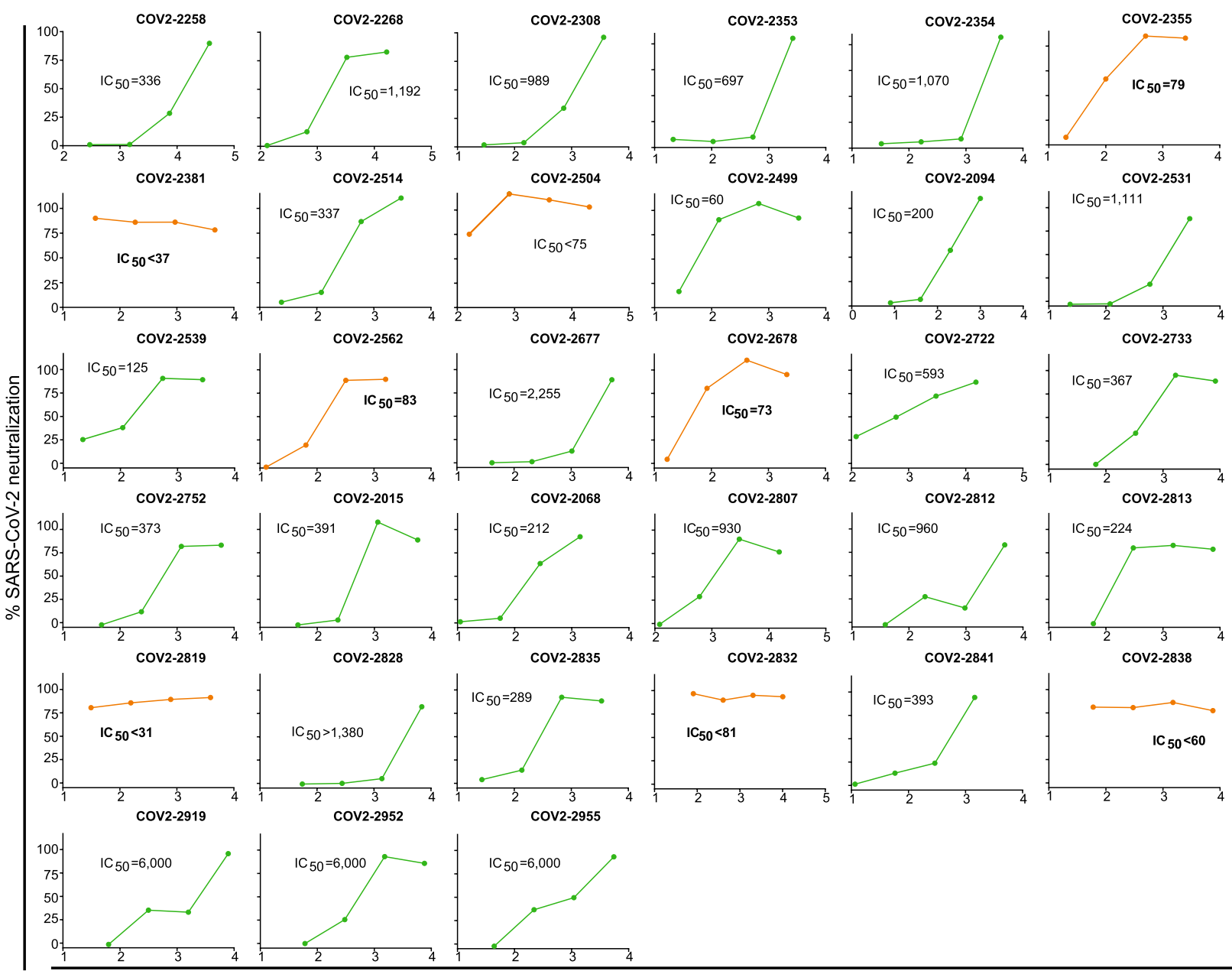

b

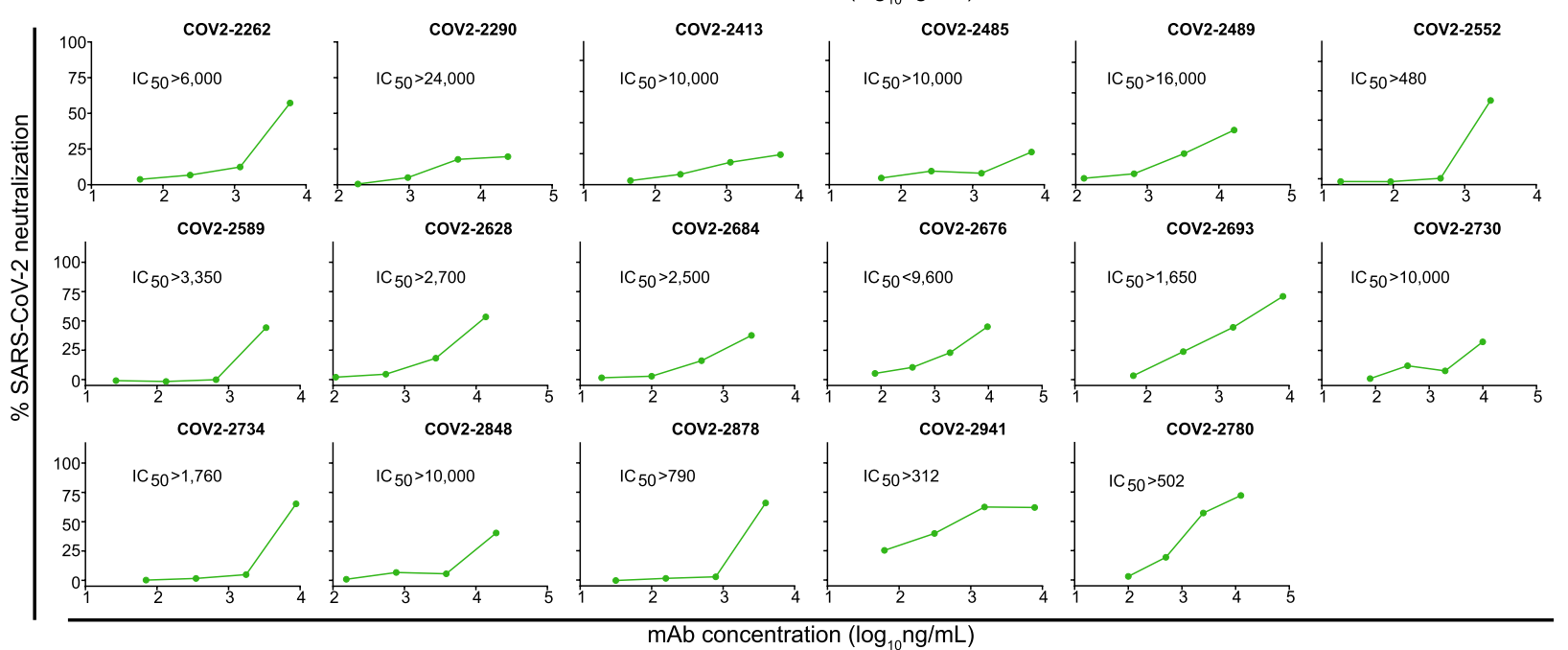

Extended Data Fig. 5 | Real-time cell analysis assay to quantify neutralization potency. Dose-response curves showing activity of neutralizing mAbs that were identified by rapid screening using the RTCA assay, as in Extended Data Fig. 4. Each mAb was tested in four sequential five-fold dilutions from micro-scale purified samples in which mAbs concentrations were not normalized but quantified. Neutralization was calculated as the percent of maximal cell index in control wells without virus minus cell index in control (virus-only) wells that exhibited maximal CPE at 40 to 48 hrs after applying virus-antibody mixture to the cells. a. Representative neutralizing mAbs that fully prevented CPE at the lowest tested dilution (corresponding to the highest tested mAb concentration) are shown. IC50 values estimated from each curve are indicated. Curves for potently neutralizing mAbs (IC50 $<100 \mathrm{ng} / \mathrm{mL})$ are shown in orange, from which mAbs COV2-2355 and COV2-2381 are genetically related. b. Representative neutralizing mAbs that partially prevented CPE at the lowest tested dilution (corresponding to the highest tested mAb concentration) are shown. 


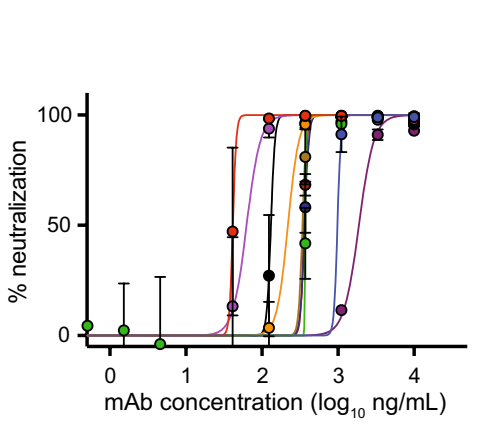

\begin{tabular}{|c|c|}
\hline $\mathrm{mAb}$ & $\mathrm{IC}_{50}(\mathrm{ng} / \mathrm{mL})$ \\
\hline - a- COV2-2015 & 983 \\
\hline- o cov2-2050 & 41 \\
\hline -o COV2-2068 & 371 \\
\hline - o- COV2-2072 & 63 \\
\hline - o- COV2-2082 & 219 \\
\hline$\rightarrow$ COV2-2094 & 131 \\
\hline -a COV2-2096 & 341 \\
\hline$\rightarrow$ COV2-2098 & 363 \\
\hline$-a-\operatorname{cov} 2-2103$ & 1,883 \\
\hline$-a-\operatorname{COV} 2-2113$ & 357 \\
\hline
\end{tabular}

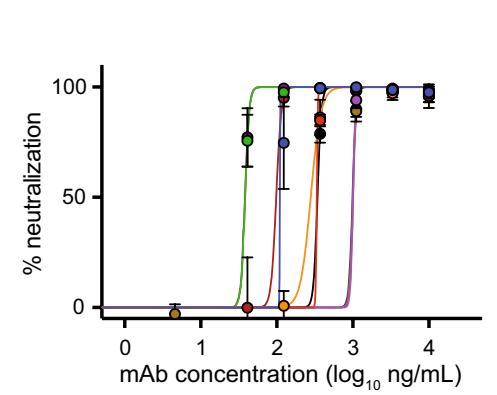

$\mathrm{mAb} \quad \mathrm{IC}_{50}(\mathrm{ng} / \mathrm{mL})$

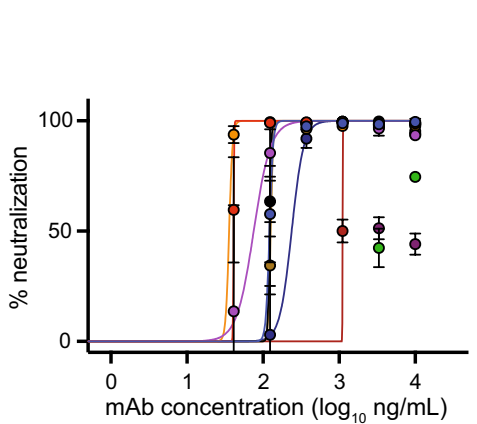

\begin{tabular}{|c|c|}
\hline $\mathrm{mAb}$ & $\mathrm{IC}_{50}(\mathrm{ng} / \mathrm{mL}$ \\
\hline - o cov2-2391 & 121 \\
\hline - cov2-2479 & 41 \\
\hline - COV2-2489 & $>3,333$ \\
\hline - cov2-2499 & 75 \\
\hline -o cov2-2504 & 36 \\
\hline$\bullet-$ Cov2-2514 & 122 \\
\hline - o cov2-2539 & 126 \\
\hline - - cov2-2562 & 238 \\
\hline - cov2-2676 & $>3,333$ \\
\hline - cov2-2677 & 1,111 \\
\hline
\end{tabular}
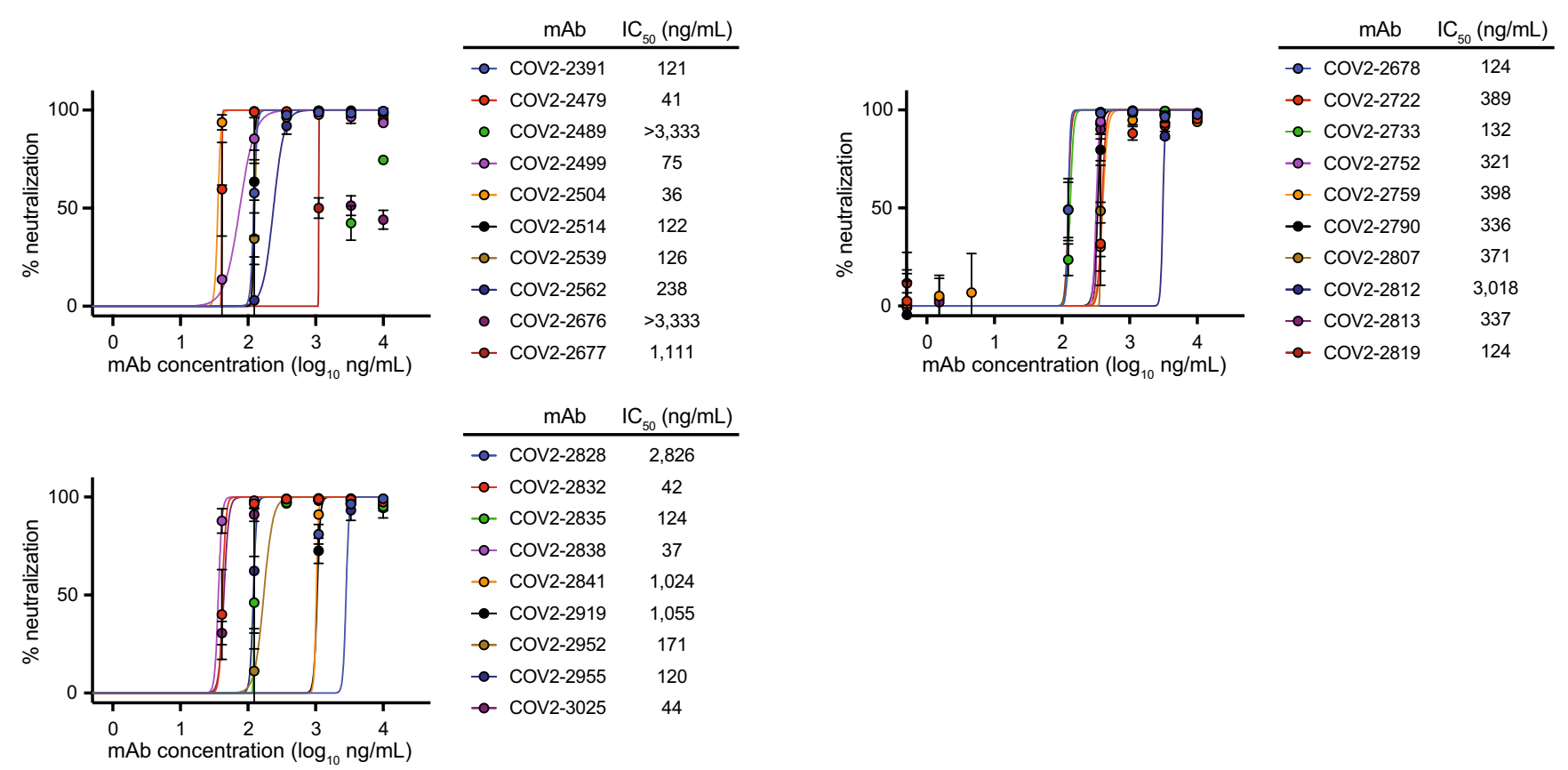

Extended Data Fig. 6 | Quantitative neutralization assays of VSV-SARS-CoV-2. Dose-response neutralization of VSV-SARS-CoV-2 by neutralizing mAbs. IC50 values are indicated for each mAb. Data shown are the mean of two technical replicates from a single experiment, and error bars denote the standard deviation for each point. 
For all statistical analyses, confirm that the following items are present in the figure legend, table legend, main text, or Methods section.

$\mathrm{n} / \mathrm{a}$ Confirmed

\ The exact sample size $(n)$ for each experimental group/condition, given as a discrete number and unit of measurement

$\square \bigotimes$ A statement on whether measurements were taken from distinct samples or whether the same sample was measured repeatedly

$\triangle \square$ The statistical test(s) used AND whether they are one- or two-sided

Only common tests should be described solely by name; describe more complex techniques in the Methods section.

$\bigotimes \square$ A description of all covariates tested

Х $\square$ A description of any assumptions or corrections, such as tests of normality and adjustment for multiple comparisons

$\checkmark$ A full description of the statistical parameters including central tendency (e.g. means) or other basic estimates (e.g. regression coefficient)

$\triangle$ AND variation (e.g. standard deviation) or associated estimates of uncertainty (e.g. confidence intervals)

$X$ For null hypothesis testing, the test statistic (e.g. $F, t, r$ ) with confidence intervals, effect sizes, degrees of freedom and $P$ value noted

Х Give $P$ values as exact values whenever suitable.

Х $\square$ For Bayesian analysis, information on the choice of priors and Markov chain Monte Carlo settings

Х $\square$ For hierarchical and complex designs, identification of the appropriate level for tests and full reporting of outcomes

$\bigotimes \square$ Estimates of effect sizes (e.g. Cohen's $d$, Pearson's $r$ ), indicating how they were calculated

Our web collection on statistics for biologists contains articles on many of the points above.

\section{Software and code}

Policy information about availability of computer code

Data collection

We used the 10X Genomics cellranger (version 2.1.1) [https://support.10xgenomics.com/single-cell-gene-expression/software/overview/ welcome] bioinformatics processing pipeline. We began by first running the cellranger mkfastq program to generate FASTQ files that were then fed into the cellranger vdj program to generate CSV and JSON files containing processed data. All processed heavy and light chain sequences were then reprocessed using our PyIR (version 1.0) [https://github.com/crowelab/PyIR] processing pipeline. PyIR is a Python wrapper that parses out VDJ assignment from IgBLAST [PMID: 23671333 ].

Data analysis

We used a customized Python script, 10x-filter.py, for downstream selection of heavy-light pairs for expression and testing. The script begins by filtering out all ambiguous heavy and light chain pairings. It then bins all unique heavy chain somatic variants with the same V3J clonotype (i.e., $V$ germline gene, J germline gene and CDR3 amino acid sequence). The heavy chain somatic variants are then rankordered within each V3J clonotype bin from most to least mutated. The user has the option to output only the most mutated sequence or least mutated sequence from each V3J clonotype bin for downstream expression and characterization. Code used for sequence processing for selecting sequences for synthesis and for analyses that appear in the paper is available at https://github.com/crowelab/ cov2-panel-scripts.

For ELISAs binding data and neutralization assays, analyses of data were performed using Prism 8.0 (GraphPad Inc).

For generation of structural schematics for Figure 3, PyMOL (Schrödinger) was used to visualize previously deposited cryo EM and crystal structures.

For IgG quantification, data were analyzed using ForeCyt software version 6.2 (IntelliCyt Corp).

For RTCA neutralization assays, sensograms were visualized and analyzed using RTCA HT software version 1.0.1 (ACEA Biosciences Inc).

For VSV-SARS-CoV-2 neutralization assays, images were analyzed using the Multi Target Analysis Module of the InCell Analyzer 1000 Workstation Software (GE Healthcare). GFP-positive cells were identified using the top hat segmentation method and counted within the 
Policy information about availability of data

All manuscripts must include a data availability statement. This statement should provide the following information, where applicable:

- Accession codes, unique identifiers, or web links for publicly available datasets

- A list of figures that have associated raw data

- A description of any restrictions on data availability

The main data supporting the results in this study are available within the paper and Supplementary Information. The ImMunoGeneTics database is available from http://www.imgt.org/. The analysis pipeline PyIR (https://github.com/crowelab/PyIR) and the specific scripts used for sequence analysis (https://github.com/ crowelab/cov2-panel-scripts) are available. Structures deposited by other groups for the full-length spike trimer (6VYB) and the RBD-hACE2 complex (6MOJ) that were used for visualization in this paper are publicly available (www.rcsb.org). Sequences for mAbs described in this study have been deposited at GenBank and are available under the following accession codes: MT665032 - MT665070, MT665419 - MT665457, MT665071 - MT665418, and MT665458 - MT665805. Datasets are available from the corresponding authors upon reasonable request.

\section{Field-specific reporting}

Please select the one below that is the best fit for your research. If you are not sure, read the appropriate sections before making your selection.

$\bigotimes$ Life sciences $\quad \square$ Behavioural \& social sciences $\quad \square$ Ecological, evolutionary \& environmental sciences

For a reference copy of the document with all sections, see nature.com/documents/nr-reporting-summary-flat.pdf

\section{Life sciences study design}

All studies must disclose on these points even when the disclosure is negative.

Sample size No sample-size calculations were performed. For mAb isolation, a large number of antibodies cloned and synthesized yielded mAbs targeting multiple epitopes on the spike glycoprotein, suggesting that the pandel described here represents a diverse repertoire from these donors. Details about research subjects groups are provided in Supplementary information.

Data exclusions No data were excluded from the analysis

Replication $\quad$ Key experimental findings that include identification of SARS-CoV2-reactive and neutralizing human monoclonal antibodies were confirmed in two or more independent experiments. Initial neutralization screening results by the RTCA assay were confirmed using a VSV-SARS-CoV-2 virus neutralization assay, and the neutralization activity of several of these mAbs was confirmed using a SARS-CoV-2 luciferase reporter virus in a BSL-3 neutralization assay. All mAbs that neutralized in the cell impedance assay showed neutralizing activity in subsequent neutralization assays.

For antigen-specific staining of PBMCs from the human subjects studied, analytical flow cytometry was performed prior to initiating processing of the larger sample. The antigen specific frequencies we observed for a given donor sample were similar across multiple days and independent staining reactions. Following sorting, a small fraction of sorted cells was analyzed by analytical flow cytometry to verify the purity of the sorted population prior to initiating single cell sequencing or B cell stimulation or expansion.

Randomization Antibody sequences were randomly allocated to different 96 well plates for DNA synthesis and for the initial antibody expression and screening assays, with the end result that antibodies with highly similar sequences and phenotypes were present across plates and experimental replicates.

Blinding The initial antibody expression and screening for antigen reactivity and neutralization activity was done in a blinded fashion, as a given antibody sequence was not known to the investigator at time of analysis. Importantly, multiple mAbs discovered by independent workflows were closely related and some had identical amino acid sequences and exhibited similar phenotypes in both antigen binding and neutralization assays. For the mAb validation experiments, investigators were not blinded to the study groups. Quantitative data analysis and validation controls were used, minimizing the risk of introducing bias through the absence of blinding.

\section{Reporting for specific materials, systems and methods}

We require information from authors about some types of materials, experimental systems and methods used in many studies. Here, indicate whether each material, system or method listed is relevant to your study. If you are not sure if a list item applies to your research, read the appropriate section before selecting a response. 


\begin{tabular}{l|l}
\hline n/a & Involved in the study \\
$\square$ & $\bigotimes$ Antibodies \\
$\square$ Eukaryotic cell lines \\
$\square$ & $\square$ Palaeontology \\
$\square$ Animals and other organisms \\
$\square$ & $\square$ Human research participants \\
$\square$ Clinical data
\end{tabular}

Antibodies used B cell phenotyping flow cytometry antibodies included APC mouse anti-human CD19 (BioLegend clone HIB19 Cat\# 982406, Lot B270238), FITC anti-human IgM (BioLegend clone MHM-88, Cat\# 314506, Lot B218736), and FITC anti-human IgD (BioLegend clone IA6-2, Cat\# 348206, lot B258195).

Polyclonal goat anti-human IgG-HRP antibody (Southern Biotech Cat 2040-05, Lot B3919-XD29) was used for antigen binding ELISA assays.

For FRNT assay, a previously described anti-SARS S-protein human antibody CR3022 (PMID: 32245784) was used as a primary antibody and the detection was performed using a goat anti-human IgG ( $\gamma$-chain specific)-peroxidase antibody (Sigma-Aldrich, Cat\# A6029).

For antigen binding and hACE2 blocking screening assays on Berkeley Lights' Beacon instrument, goat anti-human IgG $(\mathrm{H}+\mathrm{L})$ cross-adsorbed secondary antibody, Alexa Fluor 568 (ThermoFisher Scientific Cat\# A-21090), and rat anti-FLAG Alexa Fluor 647 antibody (BioLegend clone L5, Cat \#637315, Lot B265929) were used.

Validation

All antibodies used in this study except anti-S human antibody CR3022 (PMID: 32245784) are commercially available. Antibodies used in a specific species or application have been appropriately validated by manufacturers and this information is provided on their website and information datasheets as follows:

APC mouse anti-human CD19 (https://www.biolegend.com/en-us/products/apc-anti-human-cd19-antibody-14024);

FITC anti-human IgM (https://www.biolegend.com/en-us/products/fitc-anti-human-igm-antibody-2880);

FITC anti-human IgD (https://www.biolegend.com/en-gb/products/fitc-anti-human-igd-antibody-6683);

Goat anti-human IgG-HRP (https://www.southernbiotech.com/?catno=2040-05\&type=Polyclonal\#\&panel1-1\&panel2-1);

Goat anti-human IgG ( $\gamma$-chain specific)-peroxidase antibody (https://www.sigmaaldrich.com/catalog/product/sigma/a6029? lang=en\&region=US).

Goat anti-human IgG $(\mathrm{H}+\mathrm{L})$ cross-adsorbed secondary antibody, Alexa Fluor 568 (https://www.thermofisher.com/order/genomedatabase/dataSheetPdf?producttype=antibody\&productsubtype=antibody_secondary\&productld=A-21090\&version=105) Rat anti-FLAG Alexa Fluor 647 (https://www.biolegend.com/fr-ch/global-elements/pdf-popup/alexa-fluor-647-anti-dykddddktag-14979?filename=Alexa\%20Fluorreg\%20647\%20anti-DYKDDDDK\%20Tag\%20Antibody.pdf\&pdfgen=true)

Activity of newly discovered SARS-CoV-2-specific monoclonal antibodies are validated via multiple assays described in this paper.

\section{Eukaryotic cell lines}

Policy information about cell lines

Cell line source(s)

Vero E6 (CRL-1586, (American Type Culture Collection [ATCC)

Vero CCL81 (CCL-81, ATCC)

HEK293T (CRL-3216 ATCC)

Vero-furin cells were obtained from T. Pierson (NIH) and have been described previously (reference 2, Online Methods).

Expi293F (ThermoFisher Scientific, A1452)

ExpiCHO (ThermoFisher Scientific, A29127)

Authentication

Mycoplasma contamination

Commonly misidentified lines (See ICLAC register)
None of the cell lines used were authenticated

All cell lines were tested and confirmed negative for Mycoplasma contamination

None

\section{Human research participants}

Policy information about studies involving human research participants

Population characteristics

We studied five subjects in the United States, four with SARS-CoV2 exposure history and one healthy subject. Research subject demographics and SARS-CoV-2 exposure history are found in Supplemental Table 3.

SARS-CoV-2-infected subjects:

Subject 1: Male, 35 years old

Subject 2: Female, 52 years old

Subject 3: Male, 56 years old 

selected for inclusion in the study based on PCR-confirmed diagnosis with SARS-CoV-2 infection and having convalesced. There was no potential self-selection bias in recruiting patients.

Ethics oversight

The studies were approved by the Institutional Review Board of Vanderbilt University Medical Center

Note that full information on the approval of the study protocol must also be provided in the manuscript.

\section{Flow Cytometry}

\section{Plots}

\section{Confirm that:}

Х The axis labels state the marker and fluorochrome used (e.g. CD4-FITC).

Х The axis scales are clearly visible. Include numbers along axes only for bottom left plot of group (a 'group' is an analysis of identical markers).

Х All plots are contour plots with outliers or pseudocolor plots.

Х A numerical value for number of cells or percentage (with statistics) is provided.

\section{Methodology}

Sample preparation

Instrument

\section{Software}

Cell population abundance

Gating strategy
The frequency of SARS-CoV-2 spike antigen-specific B cells was enumerated from B cells pre-enriched from fresh or frozen PBMCs. B cells were labeled using a soluble recombinant S2Pecto protein produced in our laboratory and commercially available receptor-binding domain (RBD)-mouse Fc-fusion recombinant protein (Sino Biological). Functionality of antigens was validated using a conventional ACE2 binding assay. Briefly, B cells were purified magnetically (STEMCELL Technologies) and stained with anti-CD19, -IgD, -IgM, phenotyping antibodies (BD Biosciences) and biotinylated S protein. 4',6-diamidino-2- phenylindole (DAPI) was used as a viability dye to discriminate dead cells. Antigen-labeled class-switched memory B cell-S complexes (CD19+IgM-IgDSARS-CoV2 S+DAPI-) were detected with phycoerythrin (PE)-labeled streptavidin conjugate and quantified using a SH800 cell sorter (Sony). After identification of the two subjects with the highest B cell response against S2Pecto protein, target-specific memory B cells were isolated by FACS using an SH800 cell sorter (from pooled PBMCs of these subjects, after labeling of B cells with biotinylated $\mathrm{S}$ protein. These details are also found in the Methods section.

A SH800 cell sorter (Sony) was used for FACS and analytical flow cytometry studies

SH800 software and FlowJo version 10 (Tree Star Inc.).

Frequency of antigen-specific B cells ranged from 0.2 to approx. $1 \%$ of class-switched memory B cells. Antigen specificity of sorted cells was validated in functional assays after production of recombinant antibodies, which included antigen binding and virus neutralization.

PBMCs were pre-enriched for B cells using magnetic negative selection with commercial (STEMCELL Technologies) kit. Extended Data Fig. 2 indicates gating strategy for sorting of antigen-labeled class switched B cells, that included staining with phenotyping anti-CD19, anti-IgM, and anti-IgD antibodies. Pre-enriched memory B cells were first gated by forward and side scatter. Dead cells were excluded using a viability dye (DAPI). Class-switched memory B cells were gated from this viable population as CD19 + , IgM-,IgD-. The gate for the antigen-specific subset was placed based on staining of B cells isolated from a non- immune healthy donor (no exposure history to SARS-CoV-2). Specificity of antigen labeling was validated in functional assays after production of recombinant antibodies, which included antigen binding and virus neutralization. 